\title{
A study of two-dimensional flow past an oscillating cylinder
}

\author{
By H. M. BLACKBURN ${ }^{1}$ AND R. D. HENDERSON \\ ${ }^{1}$ CSIRO Division of Building, Construction and Engineering, P. O. Box 56, Highett, Vic 3190 and \\ Department of Mechanical Engineering, Monash University, Clayton, Vic 3168, Australia \\ ${ }^{2}$ Aeronautics and Applied Mathematics, California Institute of Technology, Pasadena, \\ CA 91125, USA
}

(Received 6 January 1998 and in revised form 12 November 1998)

In this paper we describe a detailed study of the wake structures and flow dynamics associated with simulated two-dimensional flows past a circular cylinder that is either stationary or in simple harmonic cross-flow oscillation. Results are examined for $R e=500$ and a fixed motion amplitude of $y_{\max } / D=0.25$. The study concentrates on a domain of oscillation frequencies near the natural shedding frequency of the fixed cylinder. In addition to the change in phase of vortex shedding with respect to cylinder motion observed in previous experimental studies, we note a central band of frequencies for which the wake exhibits long-time-scale relaxation oscillator behaviour. Time-periodic states with asymmetric wake structures and non-zero mean lift were also observed for oscillation frequencies near the lower edge of the relaxation oscillator band. In this regime we compute a number of bifurcations between different wake configurations and show that the flow state is not a unique function of the oscillation frequency. Results are interpreted using an analysis of vorticity generation and transport in the base region of the cylinder. We suggest that the dynamics of the change in phase of shedding arise from a competition between two different mechanisms of vorticity production.

\section{Introduction}

The coupling between the unsteady wake of a circular cylinder in a cross-flow and motion of the cylinder is important in structural, offshore and thermal power engineering applications. A significant feature of the cylinder-wake interaction problem for simple harmonic cross-flow oscillation is that the point in the motion cycle at which vortices are formed and released is sensitive to the frequency of cylinder oscillation. Experimental flow visualization studies, such as those of Ongoren \& Rockwell (1988), demonstrate this sensitivity for flows where the frequency of cylinder cross-flow oscillation $\left(f_{o}\right)$ is close to the natural shedding frequency of the fixed cylinder $\left(f_{v}\right)$; the timing of vortex formation (measured with respect to cylinder motion) switches phase by approximately $180^{\circ}$ over a very narrow range of forced oscillation frequencies. The evidence suggests that on each side of the switch the vortex shedding mode corresponds to the usual Kármán-street wake, at least for low motion amplitudes.

The switch also produces a change in the phase of vortex-induced forces on the cylinder, and can affect the sign of mechanical energy transfer between the moving cylinder and the flow. The change in the sign of energy transfer was first suggested by Den Hartog (1934) and has since been verified experimentally, e.g. by Blackburn \& 
Melbourne (1997). As the sign of energy transfer determines whether cross-flow oscillations will tend to increase or decrease in amplitude, the mechanics of the phase switch has implications in the study of vortex-induced vibration.

The present work deals mainly with a detailed numerical investigation of the cylinder-wake interaction for a single oscillation amplitude, but over a range of very finely spaced frequencies near the vortex shedding frequency for the fixed cylinder. The Reynolds number was set sufficiently high for the switch phenomenon to be observed at the oscillation amplitude employed. The restriction to a single amplitude and Reynolds number, and especially to two-dimensional computations, was made in order to contain the demand on computer resources while still providing useful insight.

In previous studies, such as those of Gu, Chyu \& Rockwell (1994) and $\mathrm{Lu} \&$ Dalton (1996), it has been observed that as the frequency ratio $f_{o} / f_{v}$ is increased, the onset of the switch in timing of vortex formation is associated with a contraction of the vortex formation region. This observation, while interesting, does not in itself provide a mechanism which could produce the change in timing. We propose that the underlying physical mechanism responsible for the switch is a competition between two vorticity production mechanisms, and carry out an initial investigation of this hypothesis.

\section{Dimensionless groups}

Our primary characterization of results is made in terms of the frequency ratio $F=f_{o} / f_{v}$ and amplitude ratio $A=y_{\max } / D$, where $f_{v}$ is the fixed-cylinder vortex shedding frequency, $f_{o}$ and $y$ are respectively the frequency and displacement of the simple harmonic cross-flow oscillation, and $D$ is the cylinder diameter. The instantaneous displacement is described by the dimensionless variable $\alpha(t)=y(t) / D$. Reynolds number is based on the free-stream flow speed $U$ and cylinder diameter, i.e. $R e \equiv U D / v$, and the fixed-cylinder vortex shedding frequency is described by the Strouhal number $S t \equiv f_{v} D / U$. Lift and drag forces per unit length, $F_{l}$ and $F_{d}$, are non-dimensionalized by $\frac{1}{2} \rho U^{2} D$ to give lift and drag coefficients, $C_{l}$ and $C_{d}$. The base pressure coefficient is defined as $C_{p b}=1+2\left(p_{180}-p_{0}\right) / \rho U^{2}$ where $p_{0}$ and $p_{180}$ are the pressures at the furthest upstream and downstream points on the cylinder surface.

The mechanical energy transferred from the flowing fluid to the oscillating cylinder per motion cycle can be written in dimensionless form as

$$
E=\frac{2}{\rho U^{2} D^{2}} \int_{0}^{T} \dot{y} F_{l} \mathrm{~d} t=\int_{0}^{T} \dot{\alpha} C_{l} \mathrm{~d} t=\frac{1}{2} \oint\left(C_{l} \mathrm{~d} \alpha+\alpha \mathrm{d} C_{l}\right),
$$

where $T$ is the motion period. The quantity $E$ is positive when work is done on the cylinder, negative when work is done on the fluid. A Green's theorem has been used to derive the last integral as the signed area enclosed by a limit cycle on an $\left(\alpha, C_{l}\right)$ plot, with the sense of traverse in the path integral set the same as that observed on the limit cycle.

The phase angle, $\phi$, between the cross-flow displacement $y(t)$ and the fundamental harmonic of the lift force $F_{l}(t)$ can also be used as an indicator of the transfer of mechanical energy from the fluid to the cylinder. For positive values of $E, \phi$ lies in the range $0-180^{\circ}$. 


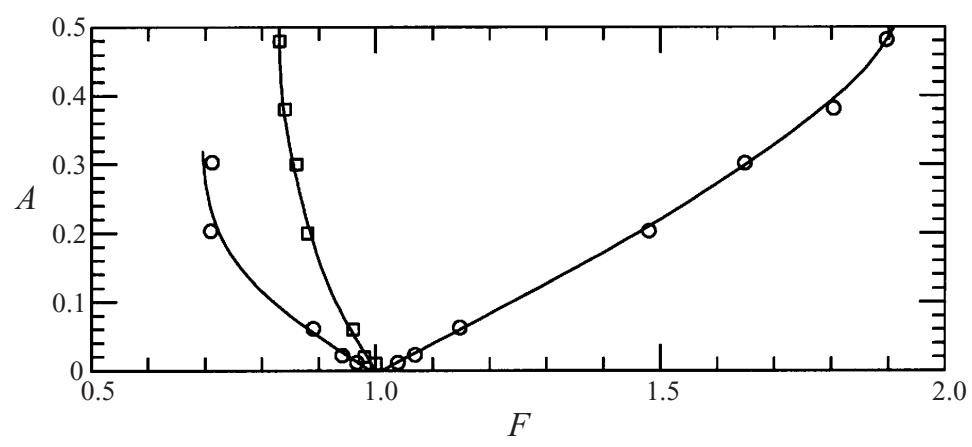

FiguRE 1. Frequency entrainment boundaries $(O)$ and wake velocity phase switch locus $(\square)$ observed by Stansby (1976) at $R e=3600$.

\section{Previous work}

Although much of the underlying motivation for forced oscillation studies obviously lies with the vortex-induced vibration problem, the bulk of what follows deals with forced oscillation studies. A review of relevant vortex-induced vibration results is given in $\S 3.3$.

\subsection{Entrainment}

The most well-known feature of the fluid-structure interaction between a circular cylinder in forced cross-flow oscillation and its wake is the primary lock-in regime. This occurs when the frequencies of vortex shedding and cross-flow oscillation coalesce near $F=1$ as the result of an entrainment process. In forced oscillation studies, vortex shedding is entrained by the cylinder motion, hence the vortex shedding frequency changes to match the cylinder oscillation frequency.

The general structure of the entrainment envelope is illustrated in figure 1 which shows results obtained by Stansby (1976) with $R e=3600$; also shown is the $(A, F)$ locus of points at which the phase angle between a wake velocity signal and cylinder motion changed by approximately $180^{\circ}$. The envelope of entrainment is thought to begin at $F=1$ for $A \rightarrow 0^{+}$, and as shown the range of $F$ expands as the oscillation amplitude increases.

Some results, such as those of Koopman (1967), suggest that a critical minimum amplitude of oscillation must be reached before entrainment occurs, and in his experiments this was $A \simeq 0.05$. In Koopman's case, it seems probable in the light of subsequent experimental results (e.g. Williamson 1989) that the value of the minimum amplitude was associated with overcoming the occurrence of slant-wise, as opposed to parallel, vortex shedding for the stationary cylinder (see e.g. figures 4 and 5 of Koopman's paper).

It is likely that some limiting minimum amplitude does indeed exist in most experimental studies, since below some small amplitude the perturbation imposed on the flow by the cylinder oscillation will be too weak to maintain control of the turbulent wake. As the cylinder wake exhibits spatio-temporal chaos immediately with the onset of three-dimensionality at $R e \simeq 190$ (Henderson 1997), a limiting minimum amplitude for continual entrainment can be presumed to exist at all higher Reynolds numbers. At very low motion amplitudes, intermittent entrainment replaces continual entrainment for turbulent wakes, as shown by Blackburn \& Melbourne (1997). Details 
of the entrainment envelope are sensitive to Reynolds number, end effects, tunnel blockage and turbulence levels.

\subsection{Influence of frequency ratio $F$ on the timing of vortex shedding}

Within the primary lock-in regime, many results suggest that the phase relationship between cylinder motion and vortex shedding is sensitive to the frequency ratio $F$. As noted above, early flow visualization results for forced cylinder oscillation obtained by Den Hartog (1934, reproduced by Zdravkovich 1982) clearly show two distinctly different timings of vortex formation with respect to cylinder motion for different values of frequency ratio $F$. From the visualization results, Den Hartog inferred a change in sign of the mechanical energy transferred from the flow to the cylinder during the motion cycle. Amplitudes and frequencies of oscillation were not recorded.

Den Hartog's flow visualization was followed by Bishop \& Hassan's (1964) measurements of lift and drag force for flow past a cylinder in forced cross-flow oscillation. Their results were obtained with a horizontal cylinder driven in the vertical direction in a water channel with a free surface. These results have had substantial impact although, as has also been noted by Bearman \& Currie (1979), difficulties associated with cancellation of inertia forces lead to some reservations about both the accuracy of their measurements and the details of their conclusions. Bishop \& Hassan's results show distinct changes in both amplitude and timing of vortex-induced forces exerted on the cylinder as the oscillation frequency is changed within the primary lock-in range. In addition, they found evidence of hysteresis, with different paths or solution branches traversed, depending on whether the oscillation frequency was incrementally increased or decreased between measurements.

Bishop \& Hassan identified two 'critical frequencies' (values of $F$ ) bounding the hysteresis loop: at $R e=6000$ and $A=0.25$, these frequency ratios were $F=0.86$ and 0.95 (by interpolation in their table 1). Negative values of $\phi$ (i.e. $E$ negative) occurred for $F$ above the upper critical value, while below the lower critical frequency ratio, $\phi \simeq 170^{\circ}$ ( $E$ positive). With $F$ decreasing from high values, the branch which started with $\phi$ negative was followed, with a gradual increase in $\phi$ until a value of 'about $90^{\circ}$ ' was reached at the lower critical frequency. At this point 'the motion is unstable and the phase angle jumps suddenly from about $90^{\circ}$ to about $180^{\circ}$, without having an intermediate value, these angles being with respect to the original direction'. On increasing $F$ from low values, the $\phi \simeq 170^{\circ}$ branch was followed until the upper critical frequency was reached, at which point the "phase angle changes suddenly by $180^{\circ}$, so there is no frequency range in which the phase angle changes gradually' (Quotations taken from $\S 9.3$ of their paper.)

Stansby (1976) conducted experiments with forced cross-flow oscillations of a circular cylinder in a low-turbulence $(0.25 \%$ intensity) wind tunnel. A hot-wire probe was used to measure velocity outside the wake, $1 D$ downstream and $2.15 D$ across from the centre of the cylinder in its mean position. Analysis of the hot-wire and cylinder displacement signals enabled assessment of entrainment and also measurements of phase angle between the wake velocity and cylinder displacement signals, as shown for example in figure 1 for measurements obtained at $R e=3600$. In the primary lock-in regime, Stansby observed that the phase angle jumped approximately $180^{\circ}$ at values of $F$ which decreased with increasing $A$. At $R e=3600$ and $A=0.25$, the value of $F$ for the switch was 0.86, similar to the lower critical value of $F$ found by Bishop \& Hassan at corresponding $A$. He also observed hysteresis associated with the jump, but only for one of two Reynolds numbers (9200) and one amplitude $(A=0.29)$. From the behaviour of the velocity signals, Stansby inferred that the jump was associated 
with a change in wake width, from being greater than that for the fixed cylinder below critical $F$, to being less than that for the fixed cylinder above critical $F$.

Bearman \& Currie (1979) carried out an experiment with forced oscillation of a circular cylinder in a water channel with a free surface. The Reynolds number $\left(2.4 \times 10^{4}\right)$ and maximum amplitude of cylinder oscillation $\left(A_{\max }=1.33\right)$ were both greater than in Stansby's experiment, and the turbulence intensity was also higher, at $5.5 \%$. Bearman \& Currie used a pressure tap located at the shoulder $\left(90^{\circ}\right)$ point of the cylinder in order to provide a signal related to the lift force. In examining the phase angle between this signal and the cylinder displacement, they found a rapid switch of approximately $130^{\circ}$ magnitude, dependent on oscillation amplitude, at values of $f_{o} U / D \simeq 5.5$, which for $S t=0.2$ corresponds to $F \simeq 0.91$. Below the critical frequency, phase angles were approximately $0^{\circ}$, while above it, phase angles were approximately $120^{\circ}$ at the lower motion amplitudes. The magnitude of the fluctuating pressure also changed substantially with the switch in phase angle, with highest values of $C_{p}^{\prime}$ at higher values of $F$. No evidence of hysteretic behaviour was reported.

Ongoren \& Rockwell (1988) presented hydrogen-bubble flow visualization results from a water tunnel flow past an oscillating cylinder at $0.85<F<1.17, A=0.13$, $R e=885$. The bubbles marked the fluid upstream of the cylinder and consequently some of the details of flows inside the near wake, immediately downstream of the cylinder, were not revealed. However, there is an obvious switch in timing of vortex formation with respect to cylinder motion between $F=0.9$ and $F=1.17$, shown in their figure 2. Analysis of velocity measurements in the near wake showed the change in phase angle between cylinder displacement and wake velocity was approximately $180^{\circ}$, and occurred near $F=1$. Flows on each side of the timing switch gave rise to Kármán vortex streets (D. Rockwell 1997, private communication).

Also in 1988, Williamson \& Roshko published visualization results for flows past an oscillating cylinder, produced using a towing tank with a computer-controlled towing carriage. Reynolds numbers varied in the range 300-1000. Flow visualization was obtained using aluminium flakes on the free surface of the water, which was pierced by the cylinder. A very wide range of $A$ and $F$ values was used, with $0.2<A<5$ and $0.33<F<5$; at low amplitudes of oscillation and in the primary lock-in zone, amplitude ratio intervals of $A=0.1$ and frequency ratio intervals of $F \simeq 0.5$ were used. A number of modes of vortex shedding were observed, leading to a map for the location of different modes in the $(A, F)$ control space.

Williamson \& Roshko labelled the different shedding modes they observed according to the number of vortex-couple pairs ('P') or single vortices ('S') shed into the wake per cylinder motion cycle. Hence the conventional Kármán-mode vortex street is called ' $2 \mathrm{~S}$ ' in their nomenclature, while another antisymmetric shedding mode was labelled '2P', for two vortex pairs shed per motion cycle. Among a number of other modes, they also found an asymmetric mode with one vortex couple and a single vortex produced per motion cycle, which they labelled ' $\mathrm{P}+\mathrm{S}$ '. This asymmetric mode had also been observed in the earlier experiments of Griffin \& Ramberg (1974) at $R e=190$, although no observations of the $2 \mathrm{P}$ mode are noted in their results.

According to Williamson \& Roshko's results, near $F=1$ and at low values of $A$, the entrainment region of the $(A, F)$ control space is occupied by $2 \mathrm{P}$ mode shedding (lower values of $F$ ) and $2 \mathrm{~S}$ mode shedding (higher $F$ ). Although they did not measure vortex-induced forces in their experiment, Williamson \& Roshko associated the phase switch observed by Bishop \& Hassan with the jump from 2S, or Kármán-mode, shedding, to the $2 \mathrm{P}$ shedding mode. Uncertainty about this conclusion remains, as conversely Bishop \& Hassan presented no flow visualization, and, as noted above, the 
change in phase of shedding observed by Ongoren \& Rockwell was not accompanied by a change in shedding mode-Kármán-mode wakes occurred on both sides of the phase switch in their experiment. Likewise, Den Hartog's flow visualizations do not reveal a change in shedding mode on either side of the large switch in phase.

$\mathrm{Gu}$ et al. (1994) reported experimental measurements of near-wake vorticity fields obtained for cylinders oscillating at $A=0.2$ and two values of Reynolds number, $R e=185$ and $R e=5000$. At $R e=185$, the distance downstream from the cylinder centreline at which large-scale clusters of vorticity were observed to roll up from the main shear layers was found to reduce with increasing values of $F$. At a fixed point in the motion cycle, the side of the cylinder at which the first vortex formed changed abruptly between $F=1.10$ and $F=1.12$. Similar behaviour was observed in ensemble-averaged results at $R e=5000$, but there the timing switch occurred between $F=0.85$ and $F=0.90$.

In a numerical study, Lu \& Dalton (1996) used a finite-difference method to simulate two-dimensional flows past an oscillating cylinder at $R e=185,500$ and 1000. At $R e=185$ and $A=0.4$, the influence of $F$ on the instantaneous vorticity contours presented by $\mathrm{Lu} \&$ Dalton was similar to that shown in the results of Gu et al. at the same Reynolds number and at $A=0.2$, in that there was a switch in timing of vortex formation; also the length of the vortex formation region decreased with increasing $F$.

\subsection{Phenomena of vortex-induced vibration}

Entrainment behaviour in vortex-induced vibration of flexible or flexibly mounted circular cylinders differs from that for forced oscillation. The primary reason is that a wider range of system dynamics is available in vortex-induced vibration, where the fluid-structure coupling can occur in both directions, as opposed to forced oscillation experiments, where the fluid motion is coupled to the body motion but not vice versa. Another significant difference is that in vortex-induced vibration, the longterm average of $E$ must remain positive to match the drain of energy to mechanical damping present in any real system.

While it is conventional in vortex-induced vibration experiments to use a cylinder with fixed structural natural frequency $f_{n}$ and observe the effects of varying the flow speed $U$, it is also possible to consider the flow speed $U$ to be fixed and consider instead the effects of varying $f_{n}$. We take this line in the discussion that follows as it fits more naturally with the approach taken in forced oscillation experiments.

As for forced oscillation, entrainment occurs when $f_{n} / f_{v} \approx 1$, although it is usually also observed that $f_{n} / f_{v}<1$ during lock-in. The entrainment frequency is a free parameter: for systems in which the structure-fluid density ratio $m / \rho D^{2}$ is high (here $m$ is the cylinder mass per unit length), the entrainment frequency tends to be near $f_{n}$, i.e. the vortex shedding frequency is entrained by the structural frequency (see e.g. Feng 1968, reproduced by Parkinson 1989). Conversely, when the density ratio is low the entrainment frequency tends instead towards $f_{v}$, as shown by Angrilli, Di Silvio \& Zanardo $\left(1972, m / \rho D^{2} \sim 1.5\right)$, i.e. the fluid oscillation has a substantial influence in setting the frequency. The cylinder may be free to move in the streamwise direction in addition to the cross-flow direction: while this is the norm in engineering applications, many experiments have allowed only cross-flow motion. As the streamwise oscillation amplitude is usually relatively small in the primary lock-in regime, it is likely that the streamwise freedom has only a minor influence.

While in principle both the density ratio $m / \rho D^{2}$ and mechanical damping ratio $\zeta$ are independent parameters in vortex-induced vibration problems, they are often 
combined in a single parameter $\zeta \mathrm{m} / \rho D^{2}$. This characterization approach becomes more valid as $m / \rho D^{2}$ increases and cylinder motion becomes more nearly simple harmonic. Significant, $O(D)$, response amplitudes typically occur when $\zeta \mathrm{m} / \rho D^{2} \ll 1$. A number of results obtained with low $\zeta \mathrm{m} / \rho D^{2}$ exhibit two (or more) distinct solution branches (e.g. Feng 1968; Brika \& Laneville 1993; Khalak \& Williamson 1996). Transition between the branches often occurs with hysteresis. Smoke flow visualization results presented by Brika \& Laneville suggest that the two highest amplitude branches are associated with different vortex shedding modes, i.e. $2 \mathrm{~S}$ and $2 \mathrm{P}$ mode shedding. This in turn suggests that the transition between the solution branches is also associated with a change of timing of vortex-induced forces with respect to cylinder motion. This was demonstrated by the results of Khalak \& Williamson (1997), who presented time series of the lift phase angle $\phi$ on the two main solution branches. As the mechanical damping was very low in these experiments $(\zeta=0.0045)$, the time-average phase angles observed for the two branches were very close to $0^{\circ}$ and $180^{\circ}$. Also, it must be the case that these average values lie in the range $0-180^{\circ}$ because of the constraint that $E$ take positive (although here small) values. A related change in timing was suggested by the earlier results of Feng, who employed a single-point pressure measurement to approximately characterize the lift force.

Occasionally the change in timing found in vortex-induced vibration has been discussed as though it were the same phenomenon as found in forced cross-flow oscillation studies (e.g. Bearman \& Currie 1979; Williamson \& Roshko 1988; Brika \& Laneville 1993). While the sets of phenomena are undoubtedly related, a switch in timing associated with a change in sign of mechanical energy transfer cannot be observed in vortex-induced vibration experiments, at least not when the vibration and shedding are completely entrained and periodic. This is due to the fact that in vortex-induced vibration only positive values of mechanical energy transfer from the flow to the cylinder can exist over the long term, while the sign of $E$ is not constrained for forced oscillation.

\section{Objective and approach}

Our objective in the present work is to study the effect of variations in frequency ratio $F$ on entrainment phenomena produced by forced cross-flow oscillation within the primary synchronization regime. Understanding this type of fluid-structure interaction is made more complicated by the difficulty of visualizing and measuring the large-scale separated flow near a moving body. More fundamentally, the flows are complex and time-varying, and there are few theoretical results available to guide interpretation. We have taken a numerical simulation approach in order to overcome the operational difficulties and better understand the physical processes involved. Our calculations are limited to two-dimensional flows at a fixed Reynolds number of $R e=500$. From the calculations we determine time-dependent forces and vorticity fields for a fixed cylinder and for oscillating cylinders as a function of the frequency ratio $F$. The study is also restricted to a single cross-flow oscillation amplitude ratio $A=0.25$ in order to reduce the demand on computer resources - the trade-off has been the capacity to investigate the effect of very small variations in $F$.

A word of explanation is needed regarding the applicability of two-dimensional results for this problem. At $R e=500$ the wake of a fixed cylinder is three-dimensional and turbulent. On the evidence that spanwise correlations of forces, wake velocities, etc. all increase with increasing cylinder motion amplitude (e.g. Toebes 1969; Novak \& Tanaka 1975) it is reasonable to suggest that the harmonic motion of a long circular 
cylinder seems to suppress three-dimensionality and produce flows that are more twodimensional than their fixed-cylinder counterparts, at least in the near-wake region. The stochastic nature of turbulent three-dimensional motions is obviously neglected in our model, but we do not believe that this is important in regard to the fundamental mechanisms driving the fluid-structure interaction, because these mechanisms are primarily two-dimensional. For the study of the mechanics of phase switch, a more important factor is the dependence on Reynolds number. The strong dissipation of flows at low Reynolds number (e.g. $R e=200$ ) inhibits the phenomenon of a phase switch with a change in sign of $E$. We have found that two-dimensional simulations at Reynolds numbers higher than approximately 400 are, however, able reproduce this basic phenomenon. It is expected that the simulations reported here for $R e=500$ will provide a guide to understanding the physics of the fluid-structure interaction and the vorticity dynamics of the near-wake for large Reynolds number.

Following details of the numerical method and associated convergence studies in $\S 5$, we present in $\S 6$ results for the two-dimensional flow past a fixed cylinder at $R e=500$ in the form of instantaneous streamlines and vorticity contour plots. Here we show that a significant feature of the near wake is a strong shear layer that forms on the base surface of the cylinder and interacts with the main shear layers during the vortex formation process. Results for frequency traverses conducted at the fixed amplitude ratio $A=0.25$ are presented in $\S 7$. Two solution branches for antisymmetric, periodic, Kármán-street wakes are followed from initial conditions generated at $F=0.875$ and $F=0.975$ which lie on each side of the phase switch described above. Two additional solution branches associated with asymmetric, periodic shedding modes were discovered during the frequency-traverse simulations, giving rise to the possibility of multi-valued solutions and hysteresis at some frequency ratios. It is found that periodic solution branches are separated at intermediate frequency ratios by a weaklychaotic regime that displays characteristics associated with relaxation oscillators. In $\S 8$ we decribe this regime and the aperiodic states that arise at frequencies above and below the main synchronization regime. In $\S 9$ we advance a hypothesis for the mechanism that underlies the phase-switch behaviour and test it by controlling one of the two vorticity production mechanisms for this flow.

\section{Computational methods}

A spectral element spatial discretization was employed in conjunction with a second-order time-splitting scheme (Karniadakis, Israeli \& Orszag 1991) in order to solve the two-dimensional incompressible Navier-Stokes equations. The solver uses a primitive-variable formulation. Vorticity, when required for presentation of results, was computed in post-processing. Details of the method, its application to two-dimensional and three-dimensional simulations and stability analyses of fixed cylinder wakes, and comparisons with experimental results are discussed in Henderson \& Karniadakis (1995), Henderson (1995), Barkley \& Henderson (1996) and Henderson (1997).

In the computational technique used for the present study, the incompressible Navier-Stokes equations are solved in a moving reference frame fixed to the cylinder

$$
\frac{\partial \boldsymbol{u}}{\partial t}=-\nabla P+N(\boldsymbol{u})+v \nabla^{2} \boldsymbol{u}-\boldsymbol{a}
$$

with

$$
\nabla \cdot \boldsymbol{u}=0
$$




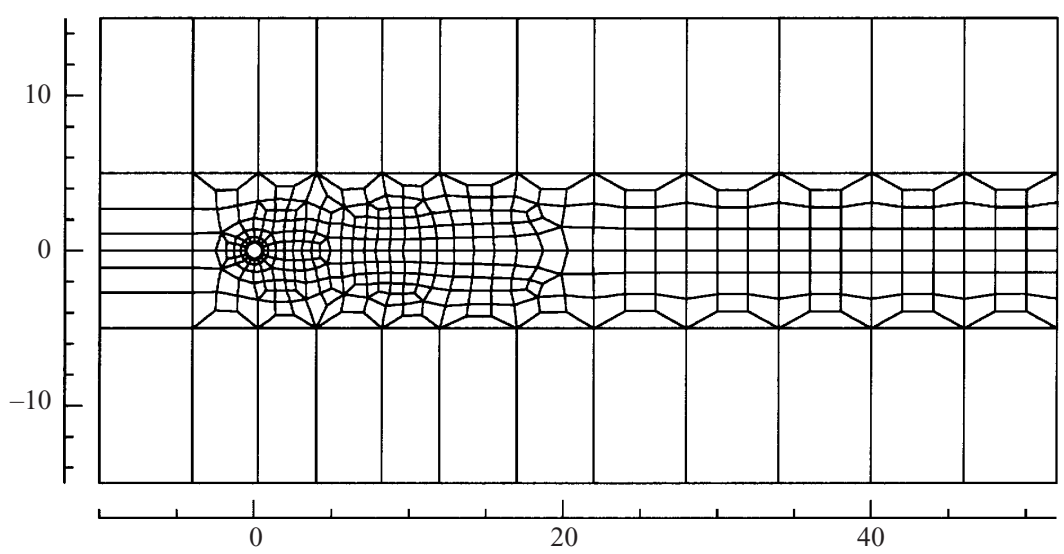

FIGURE 2. Spectral element mesh with 422 elements. Dimensions are given in terms of cylinder diameter $D$.

where $P=p / \rho, N(\boldsymbol{u})$ represents the nonlinear terms, here implemented in skewsymmetric form, i.e. $\boldsymbol{N}(\boldsymbol{u})=-(\boldsymbol{u} \cdot \nabla \boldsymbol{u}+\nabla \cdot \boldsymbol{u} \boldsymbol{u}) / 2$, and $\boldsymbol{a}$ is the acceleration of the reference frame. The boundary conditions at prescribed-velocity boundaries are set as $\boldsymbol{u}=-\boldsymbol{v}$, where $\boldsymbol{v}$ is the velocity of the reference frame. The corresponding boundary condition employed in solving the pressure Poisson equation is generated by taking the dot product of the domain unit outward normal, $\boldsymbol{n}$, with (5.1a) to give

$$
\frac{\partial P}{\partial n}=\boldsymbol{n} \cdot[\boldsymbol{N}(\boldsymbol{u})-v \nabla \times \nabla \times \boldsymbol{u}]
$$

where the form of the viscous term follows the suggestion of Orszag, Israeli \& Deville (1986). On the cylinder boundary,

$$
\frac{\partial P}{\partial n}=\boldsymbol{n} \cdot[\boldsymbol{N}(\boldsymbol{u})-v \nabla \times \nabla \times \boldsymbol{u}-\boldsymbol{a}] .
$$

The technique allows cylinder motion but maintains the shape of the mesh constant over time, avoiding computational overheads which would otherwise be associated with its geometric deformation. Previous applications of the method to both simple harmonic cylinder oscillation and vortex-induced vibration are detailed in Blackburn \& Karniadakis (1993) and Blackburn \& Henderson (1995a, $b$; 1996).

The mesh used for the calculations had 422 conforming quadrilateral elements with equal-order interpolants in all elements and is shown in figure 2 . The overall dimensions of the mesh in terms of cylinder diameter $D$ are: cross-flow 30, streamwise 62, outflow 52. A 236-element non-conforming mesh (not shown, for details see Blackburn \& Henderson 1995a) was used for cross-checking results; this mesh had overall dimensions: cross-flow 30, streamwise 65 , outflow 50 .

The results computed on the mesh shown in figure 2 have been examined for convergence by changing the order of the Gauss-Lobatto-Legendre (GLL) polynomial interpolants used (see $\S 5.1$, following), but in order to check the possible sensitivity of the results to placement and size of elements, cross-checks were carried out using the 236-element non-conforming mesh. These cross-checks were computed for single instances on each of the periodic solution branches to be described in $\S 7.3$, and while there were very minor quantitative variations in results, the computed wake vorticity contours and qualitative solution behaviours observed for the two meshes were always in good agreement. 


\begin{tabular}{ccccccc}
$p$ & \multicolumn{1}{c}{4} & \multicolumn{1}{c}{5} & \multicolumn{1}{c}{6} & \multicolumn{1}{c}{7} & \multicolumn{1}{c}{8} & \multicolumn{1}{c}{9} \\
$S t$ & 0.2287 & 0.2283 & 0.2279 & 0.2281 & 0.2278 & 0.2280 \\
$\widehat{C}_{l}$ & 1.172 & 1.171 & 1.180 & 1.198 & 1.198 & 1.200 \\
$\bar{C}_{d}$ & 1.453 & 1.452 & 1.448 & 1.461 & 1.458 & 1.460 \\
$-\bar{C}_{p b}$ & 1.478 & 1.492 & 1.487 & 1.506 & 1.504 & 1.506 \\
$\hat{u}(3.5,0)$ & 0.8833 & 0.9216 & 0.9357 & 0.9120 & 0.9242 & 0.9150
\end{tabular}

TABLE 1. $p$-Convergence results for the fixed cylinder, where $p$ is the order of the tensor-product Lagrange interpolant shape function employed in each spectral element.

$\begin{array}{ccccccc}p & 4 & 5 & 6 & 7 & 8 & 9 \\ \widehat{C}_{l} & 1.768 & 1.737 & 1.778 & 1.771 & 1.782 & 1.776 \\ \bar{C}_{d} & 1.410 & 1.396 & 1.415 & 1.412 & 1.418 & 1.414 \\ -\bar{C}_{p b} & 1.333 & 1.334 & 1.372 & 1.364 & 1.378 & 1.377 \\ \hat{u}(3.5,0) & 1.068 & 1.051 & 1.041 & 1.036 & 1.034 & 1.033\end{array}$

TABLE 2. $p$-Convergence results for the oscillating cylinder, $A=0.25, F=1.0$. St values match those for the fixed cylinder at corresponding $p$. Values of $\hat{u}(3.5,0)$ are given in the frame of reference of the oscillating cylinder.

\subsection{Convergence tests}

In order to establish appropriate choices for the order of the tensor-product GLL shape functions employed within each spectral element for the default mesh, tests were conducted both with the cylinder held fixed, and with the cylinder oscillating in cross-flow at an amplitude ratio $A=0.25$ and frequency ratio $F=1.0$. For these tests the time step was held constant at $\Delta t U / D=0.0025$.

Results of these tests are presented in tables 1 and 2, where global flow coefficients $S t, \widehat{C}_{l}, \bar{C}_{d}$ and $\bar{C}_{p b}$ are presented for different values of the order, $p$, of the polynomial basis. Here $S t$ is the Strouhal number, $\widehat{C}_{l}$ the peak coefficient of lift, $\bar{C}_{d}$ the mean coefficient of drag and $\bar{C}_{p b}$ the mean value of the coefficient of base pressure. Also included are peak values of streamwise velocity, $\hat{u}$, at a point $3.5 \mathrm{D}$ downstream from the cylinder centreline. For all measures employed the variation between the values at $p=7$ and $p=9$ was less than $1 \%$, and $p=9$ has been used for the bulk of the results reported here. Spot checks with $p=7, p=10$ and $p=11$ have also been carried out. Conversely at low values of $p$ it was found that it became impossible to obtain periodic solutions for both the cases tested: e.g. for $p=2$ for the fixed cylinder, $\widehat{C}_{l}$ would fluctuate of order $5 \%$ from cycle to cycle.

The values of $\bar{C}_{d}$ in table 1 may be compared with curve fits to two-dimensional simulation results published in Henderson (1995), where at $R e=500, \bar{C}_{d}=$ $C_{d f}+C_{d p}=0.171+1.274=1.445 ; C_{d f}$ and $C_{d p}$ are respectively the viscous and pressure contributions to the mean drag. The slight variation (approx. $1 \%$ ) between this value and the values found in table 1 gives an indication of the mesh-to-mesh variation in numerical results (primarily the result of variations in domain size) and the accuracy of the curve fit at this Reynolds number. Figure 3 shows a comparison to Henderson's curve fits for $C_{d}$, and also to three-dimensional experimental and computational results.

With the second-order time-splitting scheme, a check of temporal convergence at $p=7$ for the fixed cylinder showed that values of $S t, \widehat{C}_{l}$ and $\bar{C}_{d}$ were constant to 


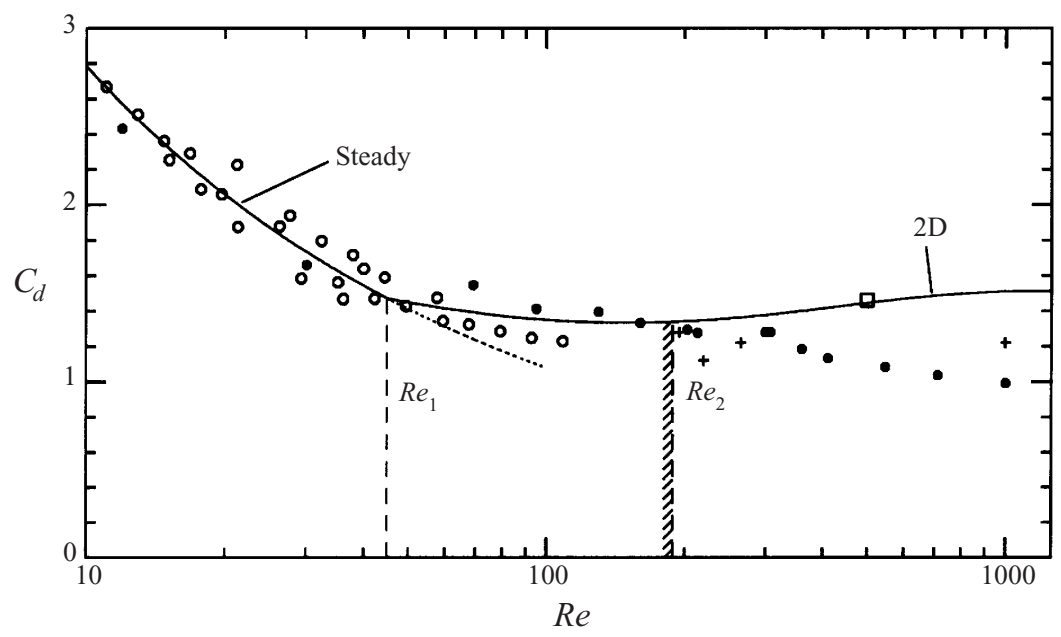

FIGURE 3. Coefficient of drag for fixed circular cylinders. •, o, Weiselsberger (1921); + , three-dimensional computations, Henderson (1997); $\square$, present results. Solid lines show curve fits to two-dimensional results of Henderson (1995). $R e_{1}$ : onset of vortex shedding, $R e_{2}$ : onset of three-dimensionality.

four significant figures over the range $0.0015<\Delta t U / D<0.0045$. For the majority of results presented here, $0.0025<\Delta t U / D<0.004$, as appropriate to maintain numerical (Courant) stability in the explicit nonlinear substep of the time-splitting scheme.

\section{The fixed cylinder}

While not the main focus of this study, vorticity contours for simulation of the twodimensional flow past a fixed cylinder are included here as a basis for comparison with similar results to be presented for the oscillating cylinder. Instantaneous streamlines are also presented, although many features of the near-wake velocity field can be deduced from the vorticity contours. Figure 4 shows vorticity contours and streamlines for five instants drawn from the half of the vortex shedding cycle during which the lift force acts in the upwards direction, starting and ending at times of zero lift. Attachment and separation points are labelled $\mathrm{A}$ and $\mathrm{S}$ respectively in figure $4(a-e)$.

Four key concepts can be used as aids to interpretation of vorticity contours. First, continuity demands that separation and (re)attachment points must occur in pairs for a two-dimensional flow. Second, in the frame of reference of the body, separation and attachment occur at points of zero surface vorticity, as may be verified by a study of figure 4. Both these concepts are dealt with in detail by Lighthill (1963). Third, in an incompressible flow, vorticity production can only occur at a solid boundary in response to wall-tangential components of pressure gradient and/or boundary acceleration. For a two-dimensional flow of a Newtonian incompressible fluid the equation for the vorticity source strength at solid walls in an inertial reference frame reads (Morton 1984; Hornung 1989):

$$
-v \boldsymbol{n} \cdot \nabla \boldsymbol{\omega}=-\boldsymbol{n} \times(\nabla P+\boldsymbol{a}),
$$

where $\boldsymbol{\omega}$ is the vorticity vector, $\boldsymbol{n}$ again is a unit wall-normal vector, $\boldsymbol{a}$ is the wall acceleration and all terms are evaluated at the wall. This equation expresses the idea that vorticity must diffuse away from a solid boundary at the same rate that it is produced by local conditions. Fourth, for flow past a body that has no angular 

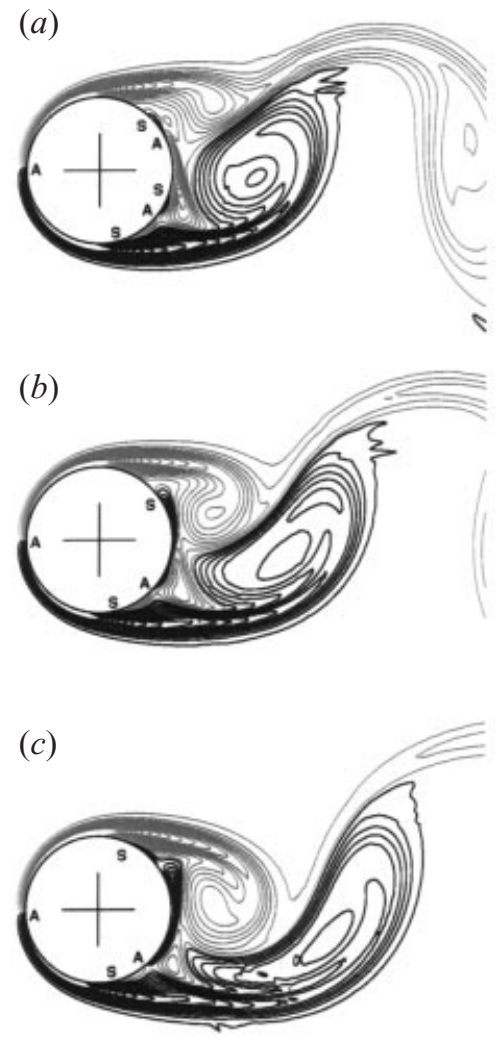

(d)

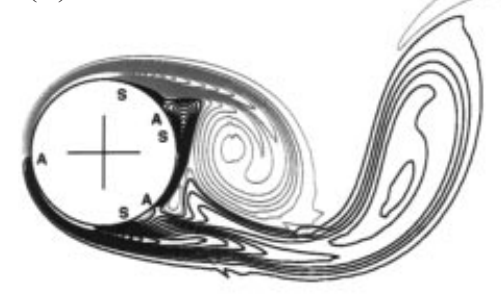

(e)

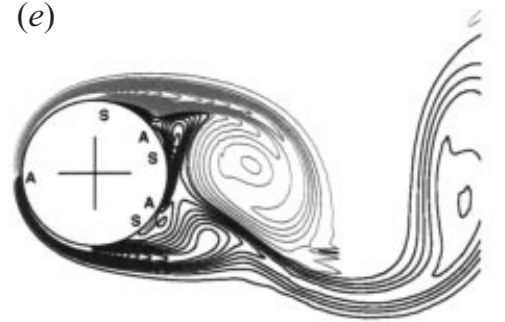

(f)

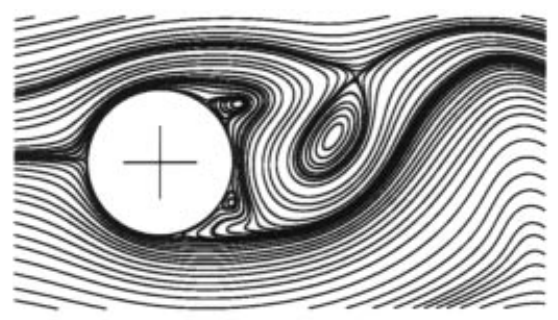

(g)

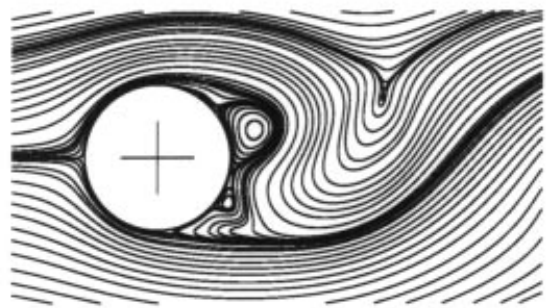

(h)

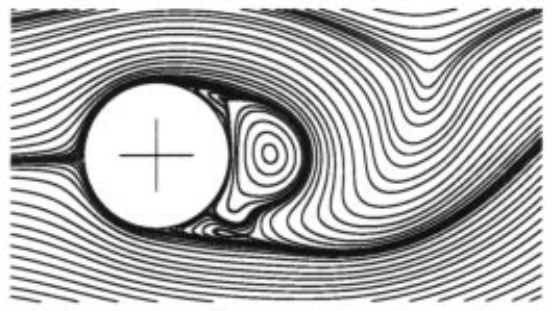

(i)

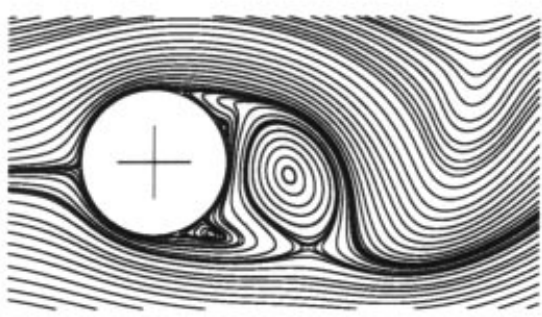

(j)

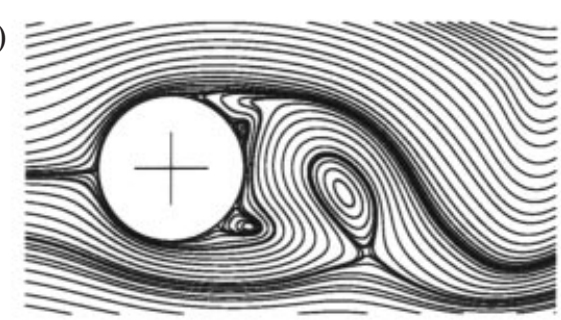

FiguRE 4. Fixed cylinder, $R e=500$. Instantaneous vorticity contours and streamlines for the half of the vortex shedding cycle during which lift is positive-lift is zero for $(a)$ and $(e)$. Black vorticity contours show vorticity of positive sign; grey contours, negative sign. 
acceleration, the integral around the body perimeter of the flux of vorticity is zero at every instant, which is a consequence of (6.1) and the continuity of the pressure field:

$$
\oint-v \boldsymbol{n} \cdot \nabla \omega \mathrm{d} S=\oint-v \frac{\partial \omega}{\partial n} \mathrm{~d} S=0,
$$

where the scalar $\omega$ represents the single out-of-plane component of the vorticity vector in a two-dimensional flow.

There are three separation-reattachment pairs shown in figure 4(a). The two pairs closest to the horizontal on the basal (i.e. rear) surface form the terminating points for two separation bubbles - regions of flow where the bounding streamline starts and ends on the surface of the body. These bubbles have greater areas than are indicated by the two small black regions of positively-signed vorticity on the basal surface, as a comparison to figure $4(f)$ reveals. Associated with this streamline topology there is a downflow in the base region of the cylinder which advects vorticity of negative sign (grey contours), produced on the basal surface of the cylinder, towards the lower shear layer. The lower shear layer has at this stage completed the formation of a large region of positively-signed vorticity, which contains a detached region of circulating flow (i.e. a closed streamline loop). This region is accelerating away from the cylinder.

In figure 4(b) the two separation bubbles have coalesced into a single larger bubble, as indicated by the coalescence of the two regions of positive vorticity on the basal surface. At this stage there are two separation-reattachment pairs. Since the region of positive vorticity on the basal surface is growing with time, it can be inferred that in figure $4(a b)$, positive vorticity is being produced on the basal surface by a downward-acting pressure gradient, in agreement with the fact that the lift is positive at this time. Simultaneously, this vorticity begins to be advected towards the upper shear layer by flow in the recirculation bubble. The transport is limited by the local shape of instantaneous streamlines within the separation bubble, although diffusive transport, normal to the contour lines, will produce significant cross-annihilation.

At the instant of figure 4(c), the upper separation point has moved nearer the top of the cylinder, and from the streamlines of figure 4(h), there is strong advective transport of basal shear layer vorticity towards the upper main shear layer. Note also the small detached streamline loop below the main separation bubble.

In figure $4(d)$, a very small region of positive vorticity appears on the upper basal surface, enveloped by negative vorticity. At the same instant as the formation of the associated separation-reattachment pair, the lower detached streamline loop previously visible in figure $4(h)$ closes on the cylinder surface, as required to maintain parity of separation and reattachment points. This bifurcation of topology also implies detachment of the vortex that evolved in figure $4(a-c)$; the streamline bounding a region of negative vorticity now forms a closed loop that does not contact the cylinder, and the vortex accelerates downstream.

Although the vortex formation process is dominated by the roll-up of the main shear layers, a significant feature illustrated in figure 4 is the formation and advection of a comparatively strong shear layer at the rear face of the cylinder. The sign of vorticity in this basal shear layer always opposes the sign of the main shear layer towards which it advects. Apart from remarks made by Shariff, Pulliam \& Ottino (1991) this feature of the very near wake has previously attracted little comment, presumably as a consequence of observational difficulties in physical experiments. As a result of the intricate folding of material lines in the base region (Perry, Chong \& Lim 1982) it becomes very difficult to make conclusions about vorticity transport based on studies of marker (dye) transport. One of the few qualitative models for the bluff-body 
vortex shedding process (Gerrard 1966) describes vortex formation solely in terms of the interaction between the main shear layers formed on the upper and lower front surfaces of the cylinder and ignores the presence of the basal shear layer altogether.

\section{Time-periodic wakes of the oscillating cylinder}

For this section of work, simulations were carried out with the cylinder forced to oscillate in cross-flow at a fixed amplitude ratio of $A=0.25$ and with a range of frequencies $f_{o}$ from approximately 0.75 to 1.05 times the Strouhal frequency for the fixed cylinder $f_{v}$, i.e. $0.75<F<1.05$. Starting from two periodic solutions with Kármán vortex streets $(\S \S 7.1,7.2)$, results of frequency traverses were computed, and two main periodic-solution branches were found to emanate from these initial points. In addition, solution bifurcations were discovered which lead to different, periodic wake configurations - and transfer between the branches occurs with hysteresis. This material is dealt with in $\S 7.3$. For all periodic results the vortex shedding frequency, as defined from the lift time series, was the same as the frequency of cylinder motion. Aperiodic solutions will be described in $\S 8$.

\subsection{Initial results with $F=0.875$ and $F=0.975$}

From previous work (Blackburn \& Henderson 1995a, b) it was known that for frequency ratios near $F=0.875$ and $F=0.975$, markedly different timing of vortex shedding with respect to cylinder motion would be observed, and these ratios were chosen for the initial simulations. These were initiated with velocity fields corresponding to periodic vortex shedding for the fixed cylinder, and terminated once periodic states were again attained.

Examples of vorticity contours for cylinders in simple harmonic cross-flow oscillation are shown in figure 5 . The two sets of results shown (figure $5 a-e, f-j$ ) are respectively for $F=0.875$ and $F=0.975$. Each set illustrates one half-cycle of cylinder motion, starting with the cylinder in its uppermost position. Although the computations were performed in a frame of reference attached to the cylinder, they are visualized here in a fixed reference frame, indicated by the cross-hairs. The change in reference frame has a significant effect on the instantaneous streamlines, but none on the contours of vorticity because $\nabla \times \boldsymbol{a}=0$. Instantaneous streamlines and separation-reattachment points are not provided, in part because they depend on the frame of reference of the observer.

When comparing the two sets of vorticity contours in figure 5 , a marked difference in the phase relationship between vortex formation and cylinder motion can be observed. Both the resulting vorticity contours, and the sense of their change with increasing $F$, are in good agreement with results presented by $\mathrm{Gu}$ et al. (1994) and Lu \& Dalton (1996), allowing for differences in $A$ and $R e$.

The change in phase relationship is here accompanied by a change in sign of $E$. As a consequence of (2.1), the sign of $E$ can be assessed by examining a phase-plane plot of cross-flow motion and lift force, which for an entrained state will form a limit cycle. The sign of power transfer is then related to the direction of traverse on the limit cycle: for positive energy transfer to the cylinder the direction of traverse must be clockwise, while for negative transfers it must be anticlockwise. A plot showing the locked states for $F=0.875$ ( $E$ positive) and $F=0.975$ ( $E$ negative) is displayed in figure 6. 
(a)

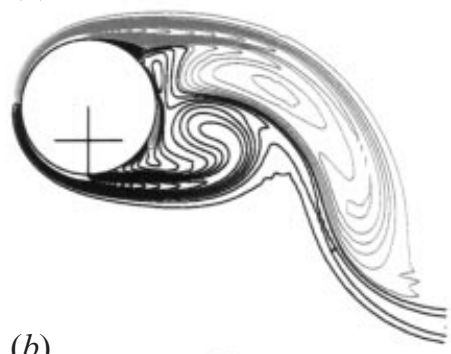

(b)

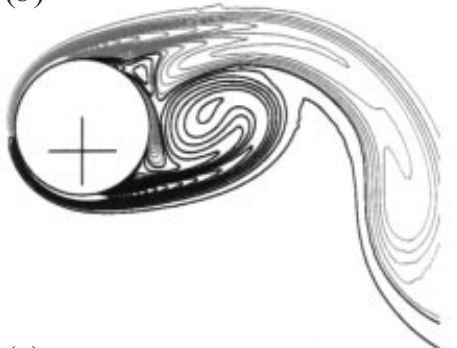

(c)

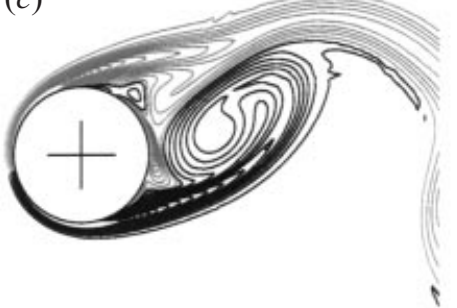

(d)

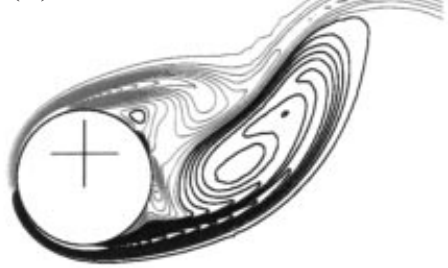

(e)

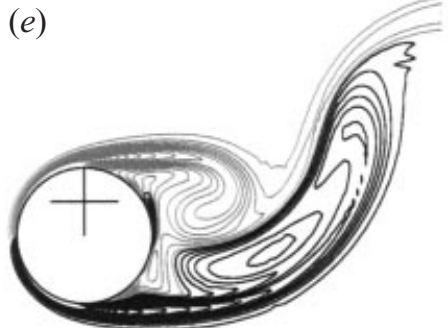

$(f)$

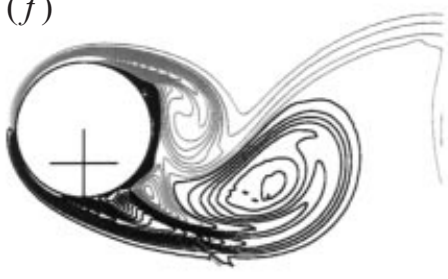

(g)

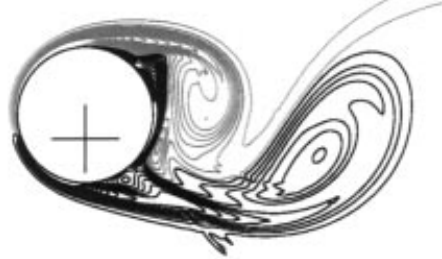

(h)

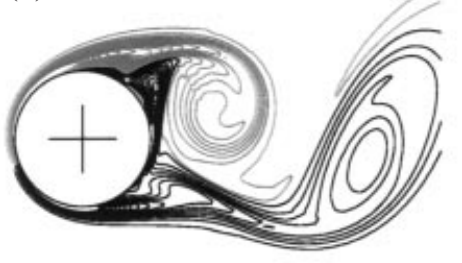

(i)

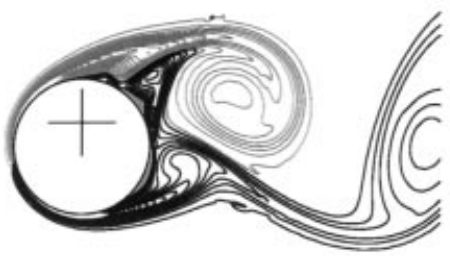

$(j)$

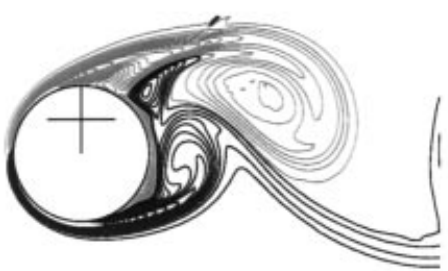

FigURE 5. Instantaneous vorticity contours over half a motion cycle for a cylinder oscillating at amplitude $A=0.25$. (a-e) $F=0.875$, energy transfer to body. $(f-j) F=0.975$, energy transfer to fluid. 


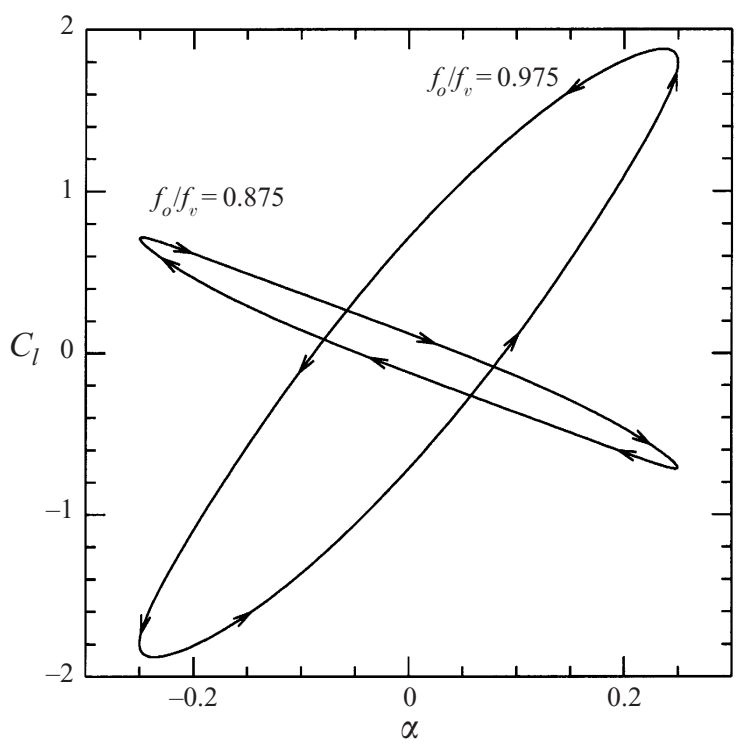

FIGURE 6. Limit cycles of coefficient of lift $C_{l}$ vs. cross-flow displacement $\alpha$ at a frequency below the phase switch $(F=0.875$, average energy transfer rate to cylinder positive) and above $(F=0.975$, transfer negative).

\subsection{Effect of initial conditions}

In order to examine the sensitivity of the solutions to the choice of initial conditions, a series of tests was conducted where the relationship between the starting time of cylinder motion and the zero-lift time of vortex shedding for the fixed cylinder were varied. The starting time $t_{0}$ for cylinder motion was set at $0,0.25 T, 0.5 T$ and $0.75 T$ relative to the zero-lift time, where $T$ is the vortex shedding period for the fixed cylinder. These tests were conducted both at $F=0.875$ and $F=0.975$. In all cases the asymptotic or long-term outcome was a periodic Kármán street wake. For $F=0.875$ the solutions reached a periodic state within a comparatively short time, $O(15)$ motion cycles. For $F=0.975$ such brief transients could also be observed but, for some values of $t_{0}$, comparatively long times, $O(200)$ motion cycles, could elapse before the periodic state was reached, as illustrated in figure 7 for the case $t_{0}=0.25 \mathrm{~T}$. During the transient, the solution changed continuously and unpredictably between cycles with positive and negative $E$; similar results are reported below in $\S 8.3$.

\subsection{Solution branches and bifurcations}

Starting with computations for the states at $F=0.875$ and $F=0.975$, two sets of frequency traverses were conducted, with frequencies both increasing and decreasing from these initial periodic solutions. The final velocity field from the previous frequency ratio solution was used to restart the computations at the next frequency ratio, with the phase angle of cylinder motion adjusted so as to match displacements between the old and new solutions. This restart technique was employed in order to mimic the behaviour that would be obtained in a physical experiment where the driving frequency of cylinder oscillation is varied in small steps. Initially, increments of frequency ratio $\Delta F=0.01$ were used, but this was varied in regions where finer increments were required.

The two solutions obtained at $F=0.875$ and $F=0.975$ both displayed wakes of 

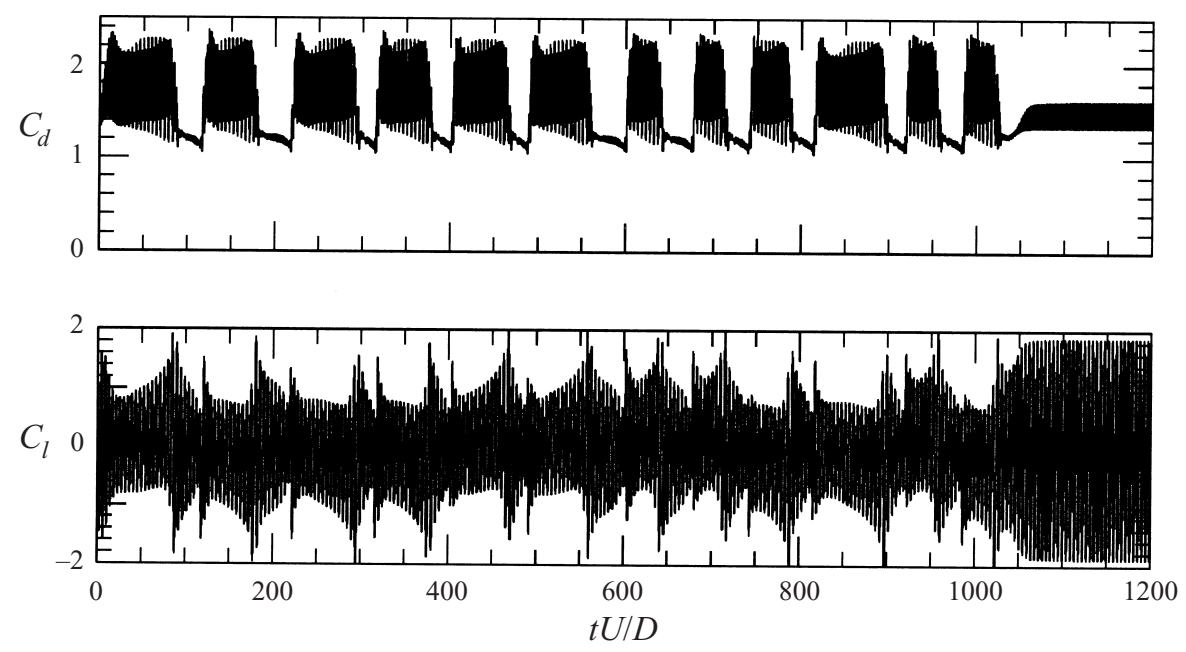

Figure 7. Time series of $C_{d}$ and $C_{l}$ at $F=0.975$, showing transient after initiation of cylinder motion.

the classic alternating Kármán street type, and the flows were perfectly periodic. As the frequencies of motion were varied by small amounts from the starting values, the solutions which evolved were also periodic after passage of sufficient time, typically on the order of 50 shedding cycles. Except for rapid transitions between solution branches, the values of $E, \widehat{C}_{l}$, etc., for each new periodic state varied smoothly on restarting and asymptotically in small increments from the previous values. Periodic solution states found in this way can each be characterized by a single variable, which in figure 8 is the dimensionless energy transfer per cycle, $E$.

In figure 8 , four solution branches can be observed, with the two longest ones $\left(K_{1}\right.$, $K_{2}$ ) being associated with Kármán street wakes, i.e. shedding modes in which two regions of concentrated vorticity of opposite sign are created during each cycle of cylinder motion, leading to an antisymmetric staggered array of vortices of opposite sign. This results in a time-mean wake which has reflection symmetry about the centreline. The two Kármán street branches are connected to the two initial states at $F=0.875$ and $F=0.975$. The two shorter branches $\left(A_{1}, A_{2}\right)$ near the upper end of the $K_{1}$ branch, however, are for periodic states with wakes which were not of the classic Kármán street type. The vortex arrays for these wakes displayed an asymmetry as opposed to the alternating antisymmetry of the Kármán street. The lack of symmetry carries over to the time-mean wake as well. In the intermediate frequency range, $0.905<F<0.95$, the flows can be characterized as weakly chaotic; at frequencies below the entrainment or synchronization band, the flows were quasiperiodic, while at frequencies above the synchronization band the flows were chaotic: all these aperiodic results are discussed in $\S 8$.

Figure 9 displays some additional data resulting from the frequency traverses. Figures $9(a)$ and $9(b)$ respectively show mean drag and peak lift coefficients normalized by the values obtained for the fixed cylinder. Figure $9(c)$ shows the phase angle $\phi$ between lift force and cylinder displacement at the frequency of cylinder oscillation. As noted in $\S 2$, positive values of $E$ correspond to phase angles between $0^{\circ}$ and $180^{\circ}$; it can be seen that the values of $F$ where values of $E$ change sign in figure 8 are the same as those where $\phi$ crosses into the range $0-180^{\circ}$. 


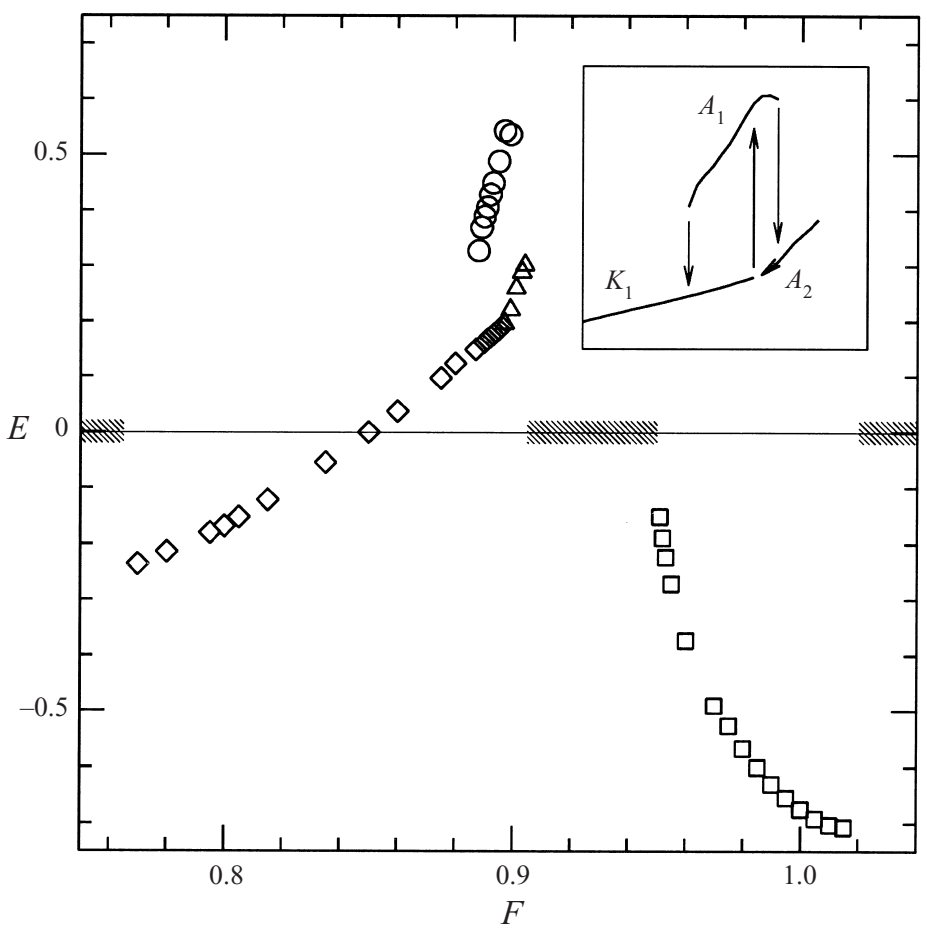

FIGURE 8. Energy transfer coefficient $E$ as a function of frequency ratio $F$ for periodic wake flows. $\diamond$, Kármán street mode, branch $K_{1} ; \square$, Kármán street mode, branch $K_{2} ; \bigcirc$, asymmetric two-cycle mode, branch $A_{1} ; \triangle$, asymmetric synchronized mode, branch $A_{2}$. Frequency ratios for aperiodic regimes are shown hatched. Inset shows paths followed in frequency traverse bifurcations.

\subsubsection{Kármán street modes}

Two Kármán street branches are shown in figure 8 . The solutions on the $K_{1}$ branch had negative values of $E$ at lower frequencies, progressing continuously to positive values at higher frequencies. The $K_{2}$ branch exhibited only negative values of $E$. Instantaneous vorticity contour plots for $F=0.80,0.875$ and 0.975 are shown in figure 10 , where plots $(a, b)$ are for solutions on the $K_{1}$ branch with negative and positive values of $E$, respectively, while plot $(c)$ is for a solution on the $K_{2}$ branch.

As will be discussed more fully in $\$ \S 8.1$ and 9 , the discontinuous transition and change in sign of $E$ between the $K_{1}$ and $K_{2}$ branches, separated by a range of frequencies in which the flow was weakly chaotic, supports a characterization of the associated change in relationship between vortex shedding and cylinder motion as a switching between different regulation mechanisms. However, the progressive change in sign of $E$ on the $K_{1}$ branch does not appear to result from such a switch.

\subsubsection{Asymmetric wake modes}

The discovery of the branches associated with asymmetric wakes occurred by chance during the frequency traverses from the two initial states. Over a band of frequency ratios approximately delineated by $0.905<F<0.95$ it was found that weakly chaotic flows resulted (\$8). Results from each frequency traverse (one with positive $\Delta F$, the other with negative $\Delta F$ ) eventually arrived again at periodic states for frequencies outside this range. That is, there was a band of intermediate frequencies over which the solution was weakly chaotic for both traverses, but outside the band solutions 

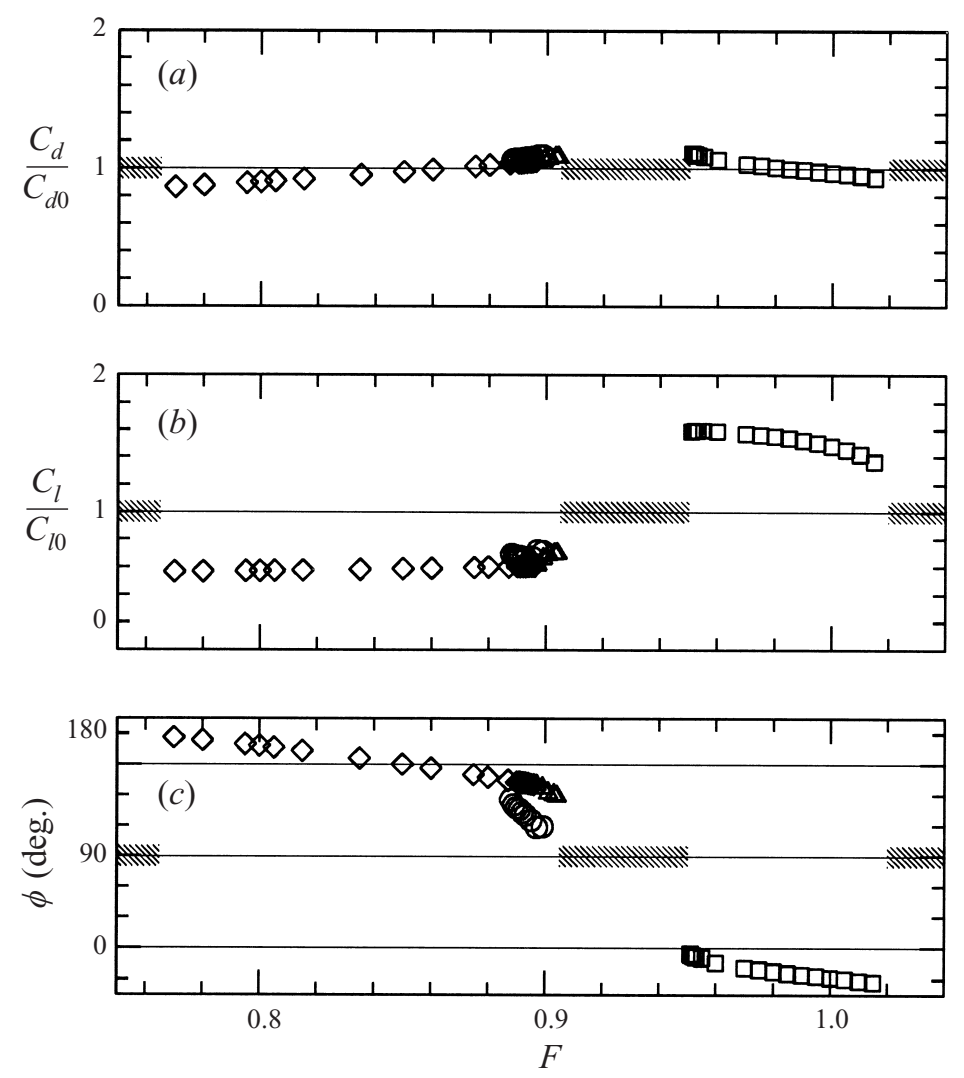

FIGURE 9. Frequency traverse results. (a) $\bar{C}_{d} / \bar{C}_{d 0}$, mean drag coefficient normalized by result for fixed cylinder. $(b) \widehat{C}_{l} / \widehat{C}_{l 0}$, peak lift coefficient normalized by result for fixed cylinder. $(c) \phi$, phase angle between lift force and cylinder displacement. Symbols $(\diamond, \square ; \bigcirc ; \triangle)$ denote the same wake modes as in figure 8 .

for each traverse again became periodic. Below the quasi-periodic range, at $F=0.89$, however, the periodic state arrived at for the negative $\Delta F$ traverse originating from the solution at $F=0.975$ was different to that obtained for the positive traverse originating at $F=0.875$.

The vorticity contours for the two states at $F=0.89$ are shown in figure 11; as in figure 5 they are shown for times when the cylinder was at the uppermost point in its motion cycle. The antisymmetric array of Kármán street wake vortices shown in figure $11(a)$ is replaced in figure $11(b)$ by an asymmetric wake. This change is reflected in the symmetry properties of the $\left(\alpha, C_{l}\right)$ limit cycles for the two cases, which are shown in figure 12. For both cases the sense of traverse on the limit cycle is clockwise, hence $E$ was positive; however the limit cycle for the asymmetric wake lacks the reflection symmetry $\left(\alpha, C_{L}\right) \rightarrow\left(-\alpha,-C_{L}\right)$ of the limit cycle corresponding to the antisymmetric wake.

The wake asymmetry in figure 11(b) results in a time-mean lift coefficient of $\bar{C}_{l}=0.066$ and is also manifested in time series of coefficients of lift and drag as a period doubling in the drag trace, but not in the lift trace, as shown in figure 13. Careful inspection of the pattern of vortices in figure 11(b) reveals that the unit of cyclic repetition of the wake array contains eight vortices, arranged as two vortex 


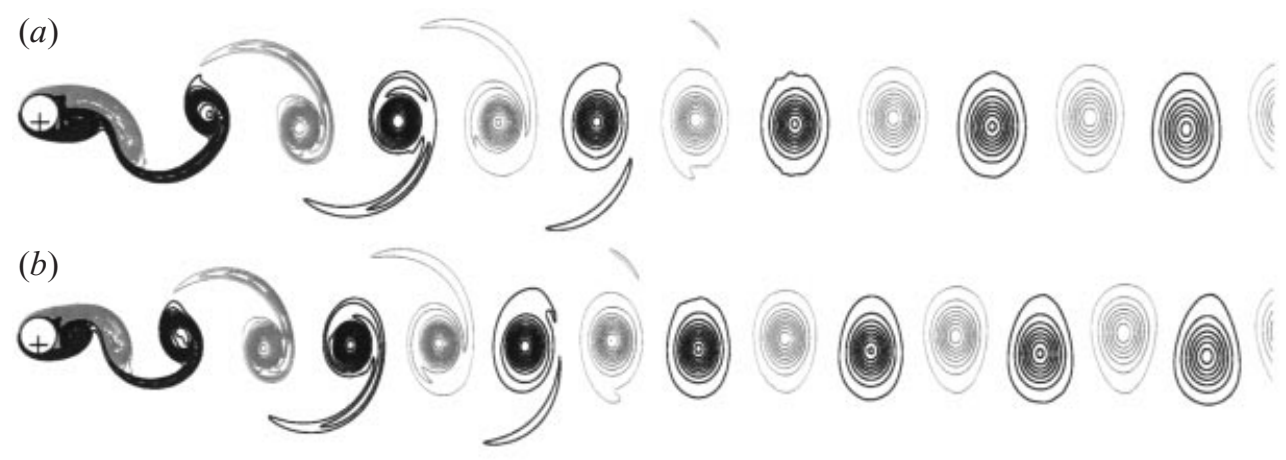

(c)

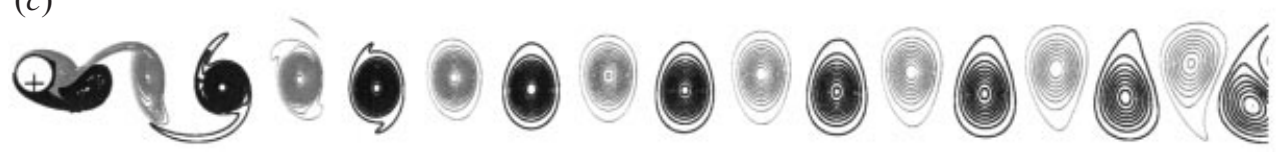

FIGURE 10. Vorticity contours for the oscillating cylinder, amplitude $A=0.25$. (a) $F=0.8, K_{1}$ branch, energy transfer to fluid. (b) $F=0.875, K_{1}$ branch, energy transfer to body. (c) $F=0.975$, $K_{2}$ branch, energy transfer to fluid. Contours are displayed for the instant in the motion cycle where the cylinder is at maximum cross-flow displacement.

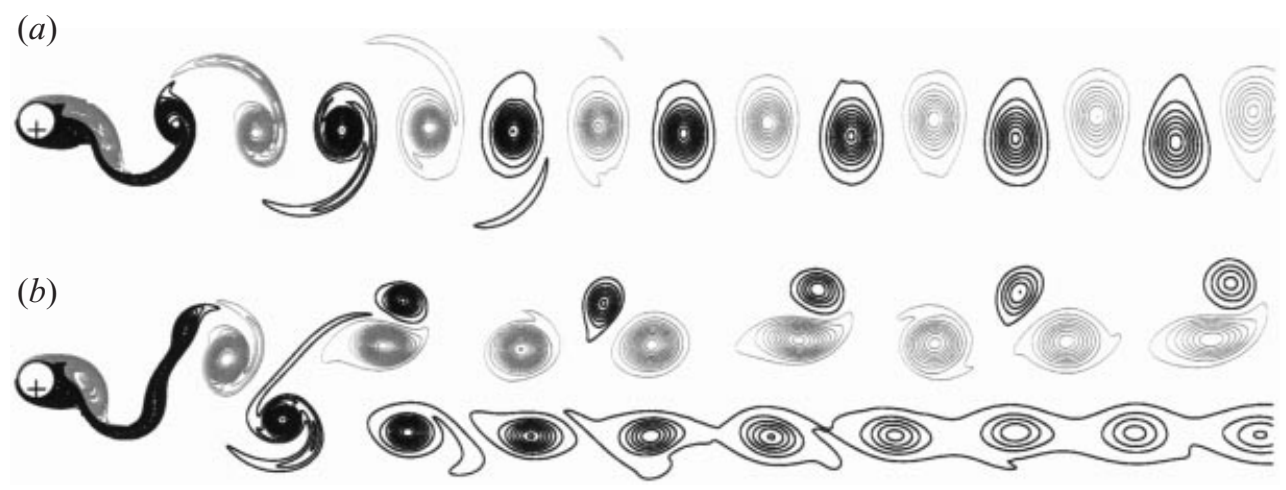

FIGURE 11. Vorticity contours for the oscillating cylinder, amplitude $A=0.25$. (a) $F=0.89, K_{1}$ branch, Kármán street mode, $(b) F=0.89, A_{1}$ branch, asymmetric two-cycle mode. Contours are displayed for the instant in the motion cycle where the cylinder is at maximum cross-flow displacement.

couples and four isolated vortices. These eight vortices are produced during two cylinder motion cycles. It is an intriguing fact that it takes two motion cycles to complete one spatial repetition of the wake array but that the $\left(\alpha, C_{l}\right)$ limit cycle is traversed in one motion cycle. We will refer to this shedding mode as the asymmetric two-cycle mode, and have labelled the associated solution branch as $A_{1}$ in figure 8 .

The discovery of the multi-valued solutions at $F=0.89$ prompted a set of investigations with finer frequency increments in order to follow the associated solution branches to higher and lower frequency ratios, as shown in figure 8 . This led to the discovery of another solution branch, $A_{2}$, with another asymmetric wake arrangement at frequency ratios just below the quasi-periodic range. Also it was found that the transitions between the different branches displayed hysteresis with respect to changes in frequency, as indicated by the arrows in the inset of figure 8 .

Vorticity contours of the wake on the second asymmetric solution branch are 


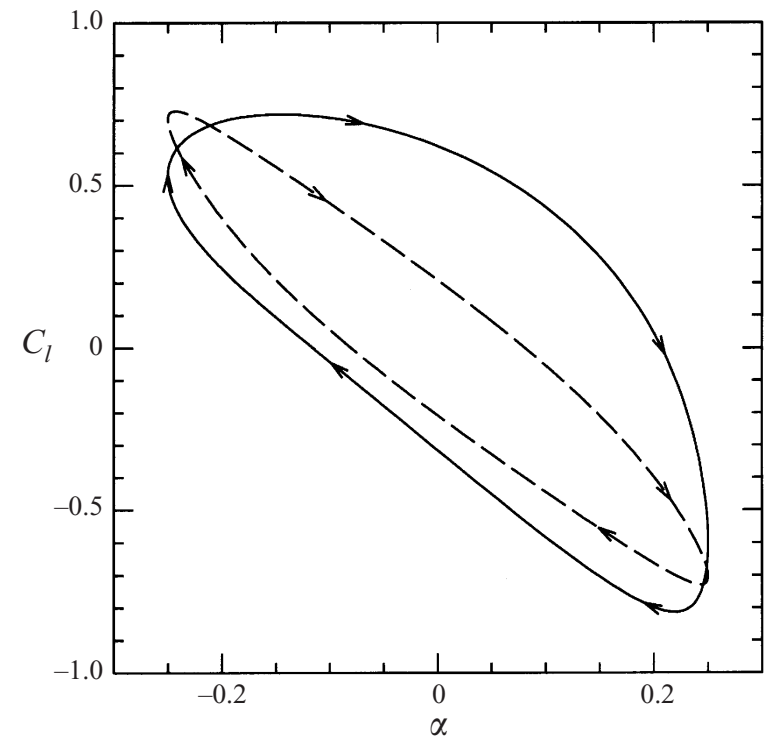

FIGURE 12. Limit cycles of coefficient of lift $C_{l}$ vs. cross-flow displacement $\alpha$ at $F=0.89$. Dashed line: $K_{1}$ branch, Kármán street mode; solid line: $A_{1}$ branch, asymmetric two-cycle mode.
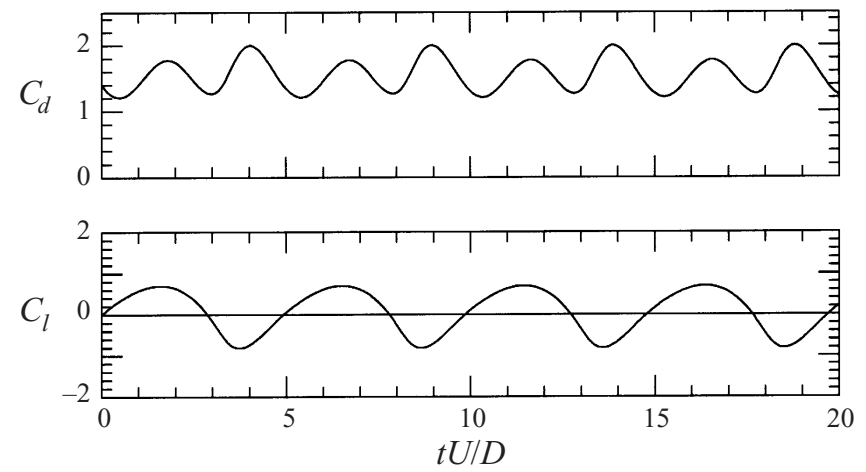

FIGURE 13. Time series of $C_{d}$ and $C_{l}$ at $F=0.89$, on the $A_{1}$ solution branch (figures 8,11 ) for the asymmetric two-cycle mode. $\bar{C}_{l}=0.066$.

shown in figure 14 , for $F=0.904$, where $\bar{C}_{l}=0.064$. The nature of the asymmetry is different to that shown in figure $11(b)$, as one cylinder motion cycle here produces a complete cycle of the arrangement of wake vortices, which evolves downstream into a vortex couple and an isolated vortex. The associated drag and lift time series are illustrated in figure 15, and again the wake asymmetry is reflected in a period-doubling of the drag trace. This mode of shedding has been reported in experimental results (Griffin \& Ramberg 1974; Williamson \& Roshko 1988); in the work of Williamson \& Roshko it was referred to as the 'P+S' (pair + single vortex) mode. Instead of this nomenclature, we will refer to it here as the asymmetric synchronized mode, since as the frequency ratio drops the wake array progressively becomes less asymmetric and eventually, prior to the transition from the $A_{2}$ branch to the $K_{1}$ branch, cannot readily be recognized as a pair and single vortex.

As noted in $\S 5$, care has been taken to confirm that the occurrence of multi-valued 


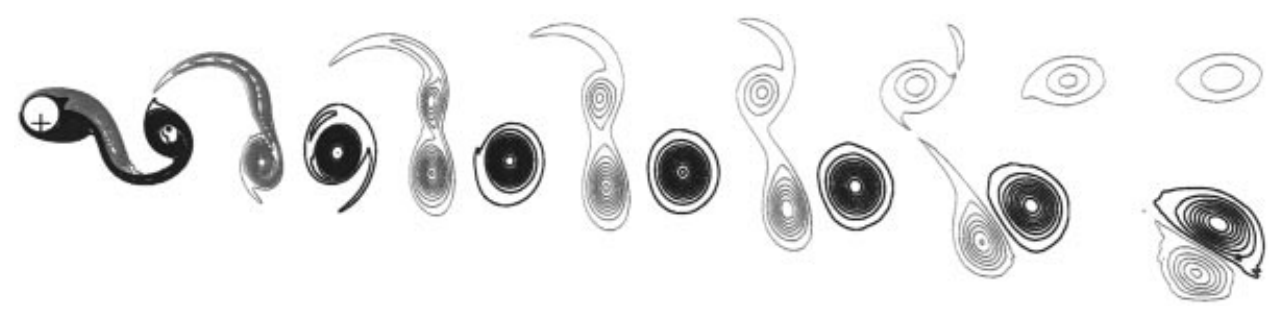

FiguRE 14. Vorticity contours for the oscillating cylinder on the $A_{2}$ solution branch (figure 8 ) for the asymmetric synchronized mode, $F=0.904$.
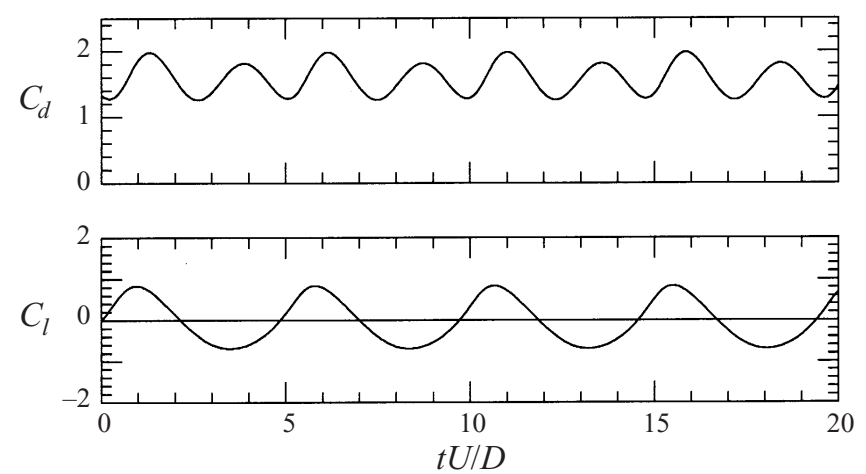

FIGURE 15. Time series of $C_{d}$ and $C_{l}$ at $F=0.904$, on the $A_{2}$ solution branch (figures 8, 14) for the asymmetric synchronized mode. $\bar{C}_{l}=0.064$.

solutions was not an artifact of numerical error in the simulations. The bifurcated states are time-periodic and reproducible over a range of solution interpolation orders, $p$, and on both the 422-element, conforming, and 236-element, non-conforming meshes. The sign of the asymmetry taken by the solution is determined by the initial conditions of the simulation. This was confirmed by the fact that the sign established during the initial transition to the $A_{1}$ branch during negative $\Delta F$ traverse from the $K_{2}$ branch was the opposite of that established as a bifurcation from the $K_{1}$ branch, while the solutions were similar in all other respects. The resulting wake vortex arrays were reflections of one another in the wake centreline, producing a change of sign of $\bar{C}_{l}$.

\subsection{Bifurcation structure}

The inset to figure 8 shows the bifurcation diagram for the $K_{1}, A_{1}$ and $A_{2}$ solution branches. The structure is made complex by the fact that the three branches are interleaved. The transitions between the $K_{1}$ and $A_{1}$ branches appear to occur through a subcritical bifurcation. Likewise the shape of the transition between the $A_{1}$ and $A_{2}$ branches suggests another subcritical bifurcation. However the linkage between the $K_{1}$ and $A_{2}$ branches is not readily categorized in terms of one-dimensional bifurcations; the fact that the $A_{2}$ branch links directly to the $K_{1}$ branch appears to indicate a supercritical-type bifurcation, but the transfer can only be made in one direction (decreasing $F$ ). In order to arrive back on the $A_{2}$ branch once the $K_{1}$ branch has been reached, transfer must first be made to the $A_{1}$ branch. This complicated linkage between the three branches indicates the presence of a higher-order bifurcation structure.

The direct connection between the $A_{2}$ and $K_{1}$ branches is also reflected in the 

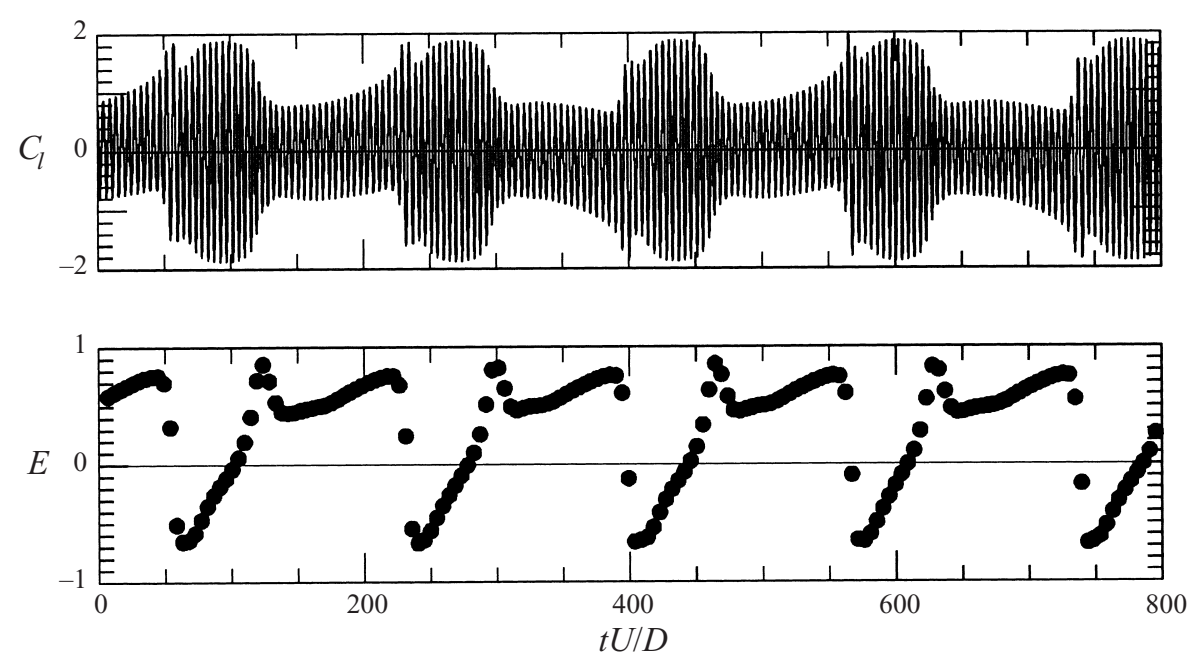

FIGURE 16. Time series of $C_{l}$ and $E$ in the intermediate weakly chaotic regime, $F=0.94$.

wake vorticity patterns. At the highest values of $F$ on the $A_{2}$ branch, the wake is asymmetric, and can be readily recognized as ' $\mathrm{P}+\mathrm{S}$ '. The degree of asymmetry reduces with $F$; as the $K_{1}$ branch is approached, the wake becomes less asymmetric, evolving to a conventional antisymmetric Kármán wake as the $K_{1}$ branch is reached.

\section{Quasi-periodic and chaotic wakes of the oscillating cylinder}

Three aperiodic flow regimes were found during the course of the investigation: $F<$ 0.77 , below the entrainment range, for which the resulting flows were quasi-periodic; $0.905<F<0.95$, where flows were weakly chaotic; $F>1.015$, above the entrainment range, for which the flows were chaotic. Our examination has concentrated on analysis of time series. We will deal with the weakly chaotic regime first, in $\S 8.1$, as it is more closely related to the periodic flows described above, before turning to the other two regimes in $\S \S 8.2$ and 8.3. We have not conducted extensive investigations of flows outside the frequency range for entrainment, so our classification of these regimes is incomplete.

\subsection{Weakly chaotic relaxation oscillator regime, $0.905<F<0.95$}

The weakly chaotic regime which was observed for $0.905<F<0.95$ was characterized by the presence of two underlying, incommensurate frequencies: one at the cylinder oscillation frequency and the other resulting from an almost-periodic switching between wake states. Fourier analysis of the lift time series in this regime shows spectra which are very sharply peaked at the cylinder oscillation frequency and its odd harmonics, but with no distinguishable peak at the long-period switching frequency. This is due to the comparatively few, $O(10)$, long-period oscillations in the time series taken for analysis. The regime could be described as quasi-periodic, as opposed to weakly chaotic, except for random selection of the sign of wake asymmetry in each of the long-period oscillations.

Figure 16 shows time series of $C_{l}$ for one of the solutions obtained in this regime, at $F=0.94$. Also shown is the time series for $E$, which emphasizes the underlying almost-periodic nature of the switching between states. At times prior to the rapid 

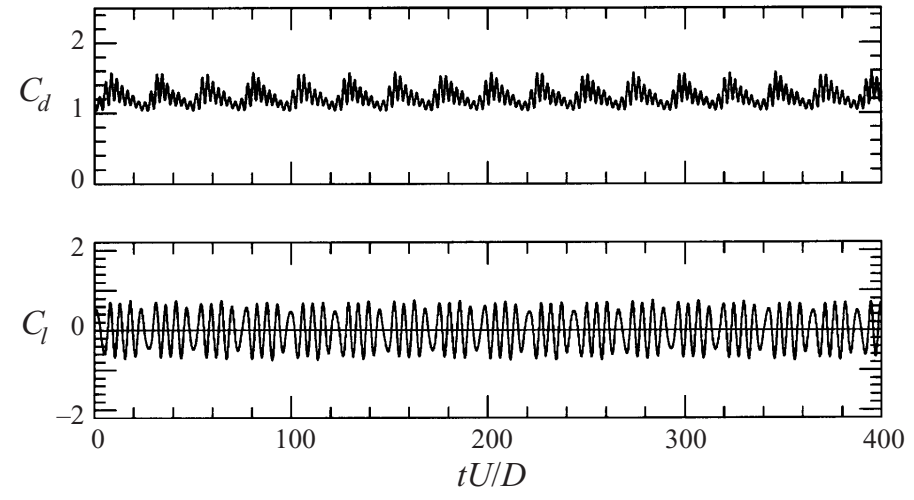

FIgURE 17. Time series of $C_{d}$ and $C_{l}$ in the quasi-periodic regime, $F=0.76$.

change in sign of $E$ at values of $t U / D \approx 50,220,390,560$ and 730, the cycleaverage values of $C_{l}$ can be seen to diverge from zero in the following sequence: ,,,,++-+- . The sequence is sensitive to initial conditions: a different sequence emerged for a simulation which was identical except for the addition of a small Gaussian-distributed perturbation to all the velocities in the initial condition. It is this sensitivity, and the associated randomness of the sequence, which indicates that the appropriate classification for this regime is weakly chaotic, as opposed to quasi-periodic.

All results obtained in this regime displayed characteristics similar to those shown in figure 16. The period associated with the switching process dropped with increasing $F$. Owing to the very long periods associated with the lowest frequency ratios, the precise nature of the variation of switching period with $F$ has not been established. At lower frequency ratios, $E$ was positive for more of the time. Also notable is the fact that the peak positive values of $E$ which can be observed in figure 16 are approximately 0.9 , much higher than the highest values observed for periodic solutions, see figure 8 .

The switching process shown in figure 16 strongly suggests relaxation oscillator behaviour, with different mechanisms vying for control of the wake dynamics. As $F$ increases, the eventual transition to a periodic Kármán street wake with small negative values of $E$ at $F>0.95$ shows that one mechanism eventually takes control as the frequency ratio increases. This theme is taken up again in $\S 9$, where we propose a hypothesis for the controlling mechanism underlying the transition between the $K_{1}$ and $K_{2}$ branches.

\subsection{Quasi-periodic solutions, $F<0.77$}

Time series of $C_{d}$ and $C_{l}$ for a flow in the quasi-periodic regime $(F=0.76)$ are shown in figure 17. Fourier analysis of the time series of lift showed very sharply peaked spectral features, with two dominant frequency components occurring at $f_{o}$ and approximately $5 f_{o} / 4$, and with additional sharp peaks at the sum and difference frequencies of these two fundamental values. Also, close inspection of the time series reveals no long-period repetition, meaning that the solution is quasi-periodic as opposed to periodic.

Similar behaviour at $F=0.75, A=0.25$ can be seen in the $R e=200$ discrete-vortex simulation results of Meneghini \& Bearman (1995, figure $9 a$ ). 

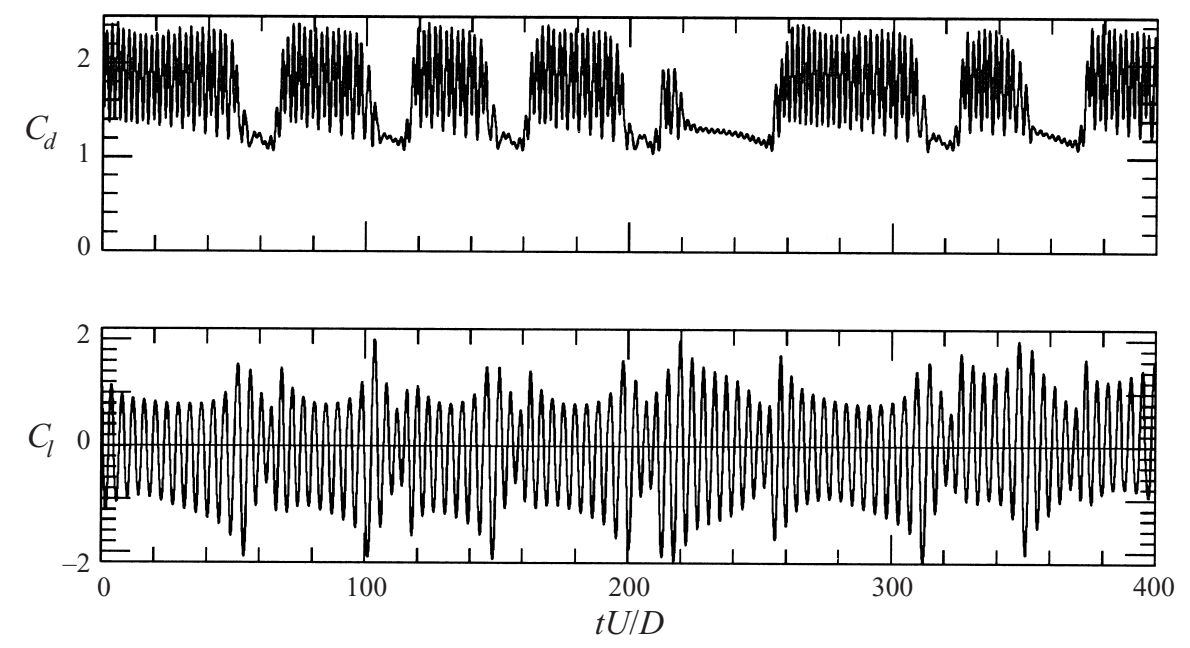

Figure 18. Time series of $C_{d}$ and $C_{l}$ in the chaotic regime, $F=1.02$.

\subsection{Chaotic solutions, $F>1.015$}

Time series of $C_{d}$ and $C_{l}$ for a flow in the chaotic regime $(F=1.02)$ are shown in figure 18. Fourier analysis of $C_{l}$ time series reveals a dominant, but not particularly sharp, peak at the Strouhal frequency, and peaks at its odd harmonics, against a background of broad-band energy. These features, together with the random nature of the time series shown in figure 18, suggest classification of this regime as chaotic.

\section{Mechanics of phase switch}

Compared to the fixed cylinder where the only mechanism for vorticity production is the tangential pressure gradient on the cylinder surface, an additional mechanism comes into play for the oscillating cylinder: the surface-tangential component of cylinder acceleration, which has maximum effect on the front and rear surfaces but none at the upper and lower points of the cylinder. According to (6.1) this motioninduced vorticity production is related to the body motion by

$$
-v \frac{\partial \omega}{\partial n}=-\boldsymbol{n} \times \boldsymbol{a},
$$

where $\boldsymbol{a}$ is the acceleration of the cylinder surface in an inertial reference frame. The net rate of production, integrated around the perimeter of the cylinder, both for this mechanism and the pressure-gradient component, is zero at each instant if the cylinder moves only as a rigid body without rotation. When the cylinder accelerates downwards (as in figures $5 a-c$ and $f-h$ ) this mechanism acts to produce fluxes of negative vorticity on the front cylinder face and positive vorticity on the rear face. Quantitatively these fluxes are of larger magnitude in figure $5(f-j)$ since the acceleration is larger than in $5(a-e)$ by the square of the ratio of the frequency ratios (a factor of 1.24).

On examination of figure 5, the formation of basal shear layers and their interaction with the main shear layers is obviously significant. In both figures $5(a)$ and $5(f)$ a region of positive vorticity near the base of the cylinder is being advected towards the upper negative vorticity-bearing shear layer. In figure $5(f)$, however, the interaction is much stronger for a number of reasons. First, the diffusive cross-annihilation must be 
far stronger, since the contour lines are more closely spaced. Second, the basal shear layer (positive vorticity) grows in strength at the same time as it is advected towards the upper main shear layer, in direct contrast to the case in figure 5(a), where positive vorticity is being annihilated in the upper base region.

\subsection{Competition between vorticity generation mechanisms}

The relaxation oscillator behaviour observed in the weakly-chaotic regime (§ 8.1) gives support to the idea that a competition between different control mechanisms underlies the discontinuous change in timing of shedding across the weakly chaotic regime. In the following we suggest a hypothesis for the mechanics of the switch.

The key feature is the relative strengths of, and competition between, the pressure gradient and motion-induced vorticity production on the basal surface. In the case of low-frequency oscillations (e.g. figure $5 a-e$ ), shear layers are perturbed such that they roll up first on the side of the wake towards which the body is moving. Pressure gradients associated with this roll-up tend to induce vorticity of opposite sign on the basal surface (as in figure $5 b, c$ ). The surface-acceleration vorticity generation mechanism opposes this production, but is not strong enough to override it.

At higher oscillation frequencies (e.g. figure $5 f-j$ ), the surface-acceleration production mechanism has been able to override the pressure gradient mechanism on the basal surface of the cylinder. A very intense basal shear layer forms, inducing earlier roll-up of the main shear layer from the opposite side of the cylinder than occurs in the lower frequency case. In turn there is a feedback effect whereby the pressure gradient produced in the roll-up of the main shear layer gives rise to vorticity production that reinforces the tangential acceleration mechanism. The strength of the main shear layer that is rolling up is enhanced, in order to achieve the balance indicated by the integral constraint (6.2).

\subsection{Simulations with reduced motion-induced vorticity production}

The relationship (9.1) can be rewritten to expose the relative contributions due to the acceleration of the reference frame attached to the cylinder and the acceleration of the cylinder surface within that reference frame:

$$
-v \frac{\partial \omega}{\partial n}=-\boldsymbol{n} \times \boldsymbol{a}=-\boldsymbol{n} \times\left(\boldsymbol{a}_{\mathrm{frame}}+\boldsymbol{a}_{\mathrm{rel}}\right) .
$$

For a given reference-frame acceleration it is kinematically possible to manipulate the tangential motion of the cylinder surface within the reference frame in order to achieve any local vorticity flux. For example if the cylinder has the prescribed motion $A D \cos 2 \pi f_{o} t \boldsymbol{j}$ then the tangential velocity required on the cylinder surface in order to reduce the motion-induced vorticity production by a factor $\beta$ at all angles $\theta$ (measured counterclockwise from the downstream direction $\boldsymbol{i}$ ) around the cylinder periphery is

$$
\boldsymbol{v}_{\text {rel }}=\beta 2 \pi f_{o} A D \sin 2 \pi f_{o} t(-\cos \theta \sin \theta \boldsymbol{i}+\cos \theta \cos \theta \boldsymbol{j}) .
$$

The motion-induced vorticity production is zero when the control parameter $\beta=1$. Such a distribution of cylinder-surface velocities would be difficult to realize experimentally, for it requires that the surface has a local tangential velocity that is a continuous but non-constant function of angular position.

In order to test the hypothesis that the controlling feature in the phase switch is the relative importance of pressure-gradient and motion-induced vorticity production, we have carried out simulations in which the motion-induced vorticity production was 
lowered artificially in the manner indicated by (9.3). No modification is required to the numerical solution technique, other than to impose a set of time-varying velocity boundary conditions on the cylinder surface. Since the velocities are tangential, there is no direct interaction with the pressure through the Neumann boundary condition (5.3).

Starting from the Kármán wake solutions obtained for $F=0.875$ and $F=0.975$ (as for figure 5), the relative motion of the cylinder surface was gradually increased from rest, by increasing $\beta$ from 0 to 1 over a period $T U / D=100$ until the motioninduced vorticity production was zero everywhere on the circumference. This resulted in the $F=0.875$ solution changing to another with a periodic Kármán street wake, and an increased value of $E$. In contrast, the periodicity of the $F=0.975$ solution was destroyed by this procedure, leading to a chaotic wake flow. Subsequently it was discovered that a periodic solution could also be obtained at $F=0.975$ if the motion-induced vorticity production was reduced by a lesser amount. With $\beta_{\max }=0.5$ this produced a Kármán street wake, with a negative value of $E$, similar in magnitude to that for the cylinder with the fixed surface at the same value of $F$.

From these two new initial periodic solutions, frequency traverses were carried out, leading to the results illustrated in figure 19. The general similarity of figure 19 to figure 8 is notable, although only positive values of $E$ are now observed on the $K_{1}$ Kármán-mode branch. Again it can be seen that multi-valued solutions were obtained for the lower- $F$, positive- $E$ regime. One of these solution branches was for antisymmetric Kármán street wakes (i.e. a $K_{1}$ branch), the other, with slightly higher $E$ values, was associated with asymmetric wakes requiring two cylinder motion cycles for one repetition of the wake vortex array (an $A_{1}$ branch). No asymmetric, synchronized $\left(A_{2}\right)$ branch was found.

\section{Discussion}

\subsection{Phenomena of the entrainment regime}

The central feature of our results is the simulation of the switch in timing of vortex formation over a narrow band of frequencies, as observed in many experimental studies (see $\S 3$ ), and the demonstration that this was accompanied by a change in sign of energy transfer between the cylinder and the fluid, as originally proposed by Den Hartog (1934). This encourages comparison with experimental results for oscillating cylinders, although, as we have noted in $\S 4$, there are some difficulties in directly relating our two-dimensional simulation results to the three-dimensional wake flows which exist at the Reynolds numbers operative for most of the experiments.

Conversely, since our simulations are two-dimensional, at a single oscillation amplitude and a fixed value of $R e$, it is unlikely that all of the wake behaviours that could occur in physical experiments have been revealed. Also it is possible that we have not found all the observable shedding modes even for the idealized case we have studied, especially as near the region of hysteresis behaviour the choice of final flow state is dependent on initial conditions.

\subsubsection{Entrainment envelope}

Our results give the frequency limits for entrainment at $A=0.25$ as $0.77<F<$ 1.015. At the same amplitude ratio, experimental results of Koopman (1967) give approximately $0.85<F<1.15$ over the Reynolds number range $100-300$, and those of Stansby (1976) give $0.71<F<1.55$ at $R e=3600$, see figure 1 . The lower limit for entrainment in our results, $F=0.77$, is then within the bounds of these two sets 


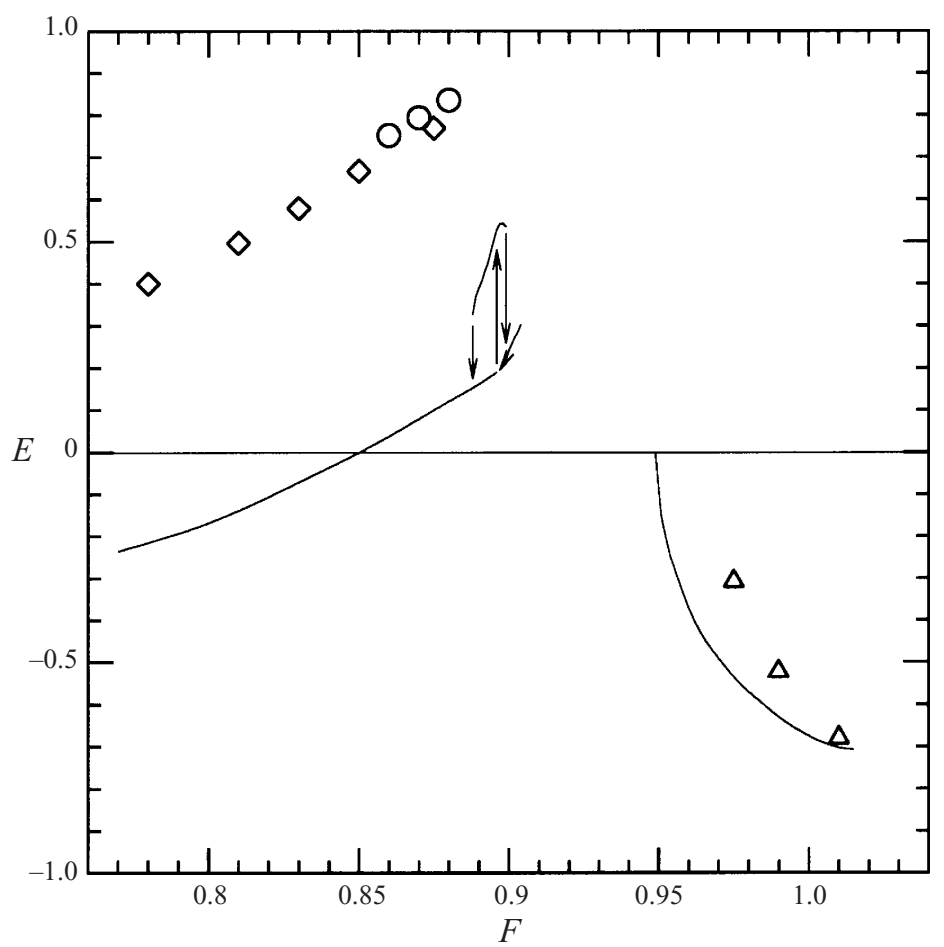

FIGURE 19. Reduced motion-induced vorticity production results. Energy transfer coefficient $E$ as a function of frequency ratio $F$ for periodic wake flows. $(\diamond, \bigcirc), \beta=1$, zero motion-induced vorticity production. $\diamond, K_{1}$ branch, Kármán mode; $\bigcirc, A_{1}$ branch, asymmetric two-cycle mode. $\triangle, \beta=0.5$, $K_{2}$ branch, Kármán mode. Solid lines indicate solution branches from figure 8.

of results, while the upper limit, $F=1.015$, is somewhat lower. According to our experience (\$7.2), at higher $F$ values the times taken to achieve periodicity could be significant and were much more sensitive to choice of initial conditions than was the case for the lower-frequency solutions. This sensitivity is perhaps reflected in variations in the high- $F$ limit for the experimental results, although these could also result from Reynolds number effects.

\subsubsection{Solution branches, hysteresis effects}

The traverse along the $K_{1}$ branch with increasing $F$ was accompanied by an apparent shortening of the vortex formation region in the near-wake (see figure 10), as has been recorded previously by Gu et al. (1994) and Lu \& Dalton (1996). Also note that the apparent width of the wake was substantially less on the $K_{2}$ branch than on the $K_{1}$ branch, as assessed by the cross flow extent of vorticity-bearing fluid in the first $10 \mathrm{D}$ of the wakes shown in figure 10 . This accords with the report by Stansby (1976, noted in §3.2) of a change in wake width on crossing the 'critical' value of $F$. Finally, the jump in $\widehat{C}_{l} / \widehat{C}_{l 0}$ values on moving from the $K_{1}$ branch to the $K_{2}$ branch (see figure $9 b$ ) agrees with a jump in $C_{p}^{\prime}$ values reported by Bearman \& Currie (1979).

There is qualitative agreement between the lift phase angles recorded by Bishop \& Hassan (1964, see the discussion in $\S 3.2)$ and values of $\phi$ shown in figure $9(c)$ for frequency ratios $F$ above and below the boundaries of the weakly-chaotic relaxation 
oscillator regime. Their upper critical frequency ratio $(F=0.95$ at $A=0.25)$ agrees exactly with the value where the $K_{2}$ solution branch terminates in our results, while their lower critical frequency ratio $(F=0.86)$ is somewhat below the lower limits of both the weakly-chaotic regime and the multi-valued solution branches.

Bishop \& Hassan's description of hysteresis behaviour can also be cast in a light that suggests some qualitative agreement with our experience. In their experiments, a solution branch with values of $\phi \simeq 90^{\circ}$ was encountered in frequency traverses with decreasing $F$. While in our simulations it was possible to transfer to a solution branch with similar values of $\phi\left(A_{1}\right)$ with increasing $F$, this bifurcation path could be bypassed if large values of $\Delta F$ were used. Thus, with reference to figure $9(c)$, a possible interpretation is that, when increasing $F$, Bishop \& Hassan's results followed the branch with highest $\phi$ values $\left(K_{1}\right)$, jumping discontinuously to the branch with negative $\phi$ at higher $F\left(K_{2}\right)$. Then while traversing with decreasing $F$, they started on the $K_{2}$ branch, encountered the $A_{1}$ branch, finally reverting to the $K_{1}$ branch.

\subsubsection{Shedding modes}

At the oscillation amplitude $A$ used in our simulations, the entire synchronization range is covered in the results of Williamson \& Roshko (1988) by either the 2P mode (at lower values of $F$ ) or the $2 \mathrm{~S}$ mode (at higher values of $F$ ), although they stated that the $\mathrm{P}+\mathrm{S}$ mode (which they included on their map but only for $A>1$ ) replaced the 2P mode for $R e<300$. While our asymmetric synchronized mode would be identified with the $\mathrm{P}+\mathrm{S}$ mode, we have not observed the $2 \mathrm{P}$ mode as a periodic solution in our simulations. It is possible that similar vortex patterns may have appeared during transient solutions, or at points in time within the quasi-periodic relaxation oscillator regime.

Another obvious point of divergence between our results and those of Williamson \& Roshko is that the mode we have called the asymmetric two-cycle mode $(2 \mathrm{P}+4 \mathrm{~S}$ in their nomenclature) was not noted at all by Williamson \& Roshko. They did observe a $2 \mathrm{P}+2 \mathrm{~S}$ mode, associated with a $1 / 3$ subharmonic entrainment, which occurs at much lower values of $F$ when $A=0.25$. It is possible however that our asymmetric two-cycle mode could evolve to the $2 \mathrm{P}$ mode at higher oscillation amplitudes, or under the influence of three-dimensional wake effects.

As noted in $\S 3.2$, only Kármán-mode shedding has been observed in some forced oscillation experiments, particularly those for low oscillation amplitudes, such as those of Ongoren \& Rockwell (1988) and Gu et al. (1994). Periodic solutions with Kármánmode shedding occupy most of the entrained frequency range in our simulation results.

A wider range of dynamical behaviour is possible in vortex-induced vibration as compared to flows with forced cylinder oscillation; however as pointed out in $\S 3.3$, only flows with positive mean values of $E$ will be observed. This implies e.g. that flows from the $K_{2}$ solution branch shown in figure 8 would not be observed in vortexinduced vibration experiments, but does not preclude coupling with flows similar to those described in the relaxation oscillator regime $(\S 8.1)$, provided $E$ is positive on average.

\subsection{Phase switch mechanics}

Our hypothesis, outlined in $\S 9.1$, is that a competition between vorticity production mechanisms is responsible both for the onset of the weakly chaotic relaxation oscillator regime and also for the discontinuous switch in phase of vortex shedding across that 
regime with increasing $F$. By manipulating the surface velocity of the cylinder, we were able to change the motion-induced vorticity production, as discussed in $\S 9.2$.

The hypothesis is supported by the fact that setting $\beta=1$ in (9.3) (i.e. reducing the motion-induced vorticity production to zero) destroyed the $K_{2}$ branch while increasing $E$ values on the $K_{1}$ branch. It is interesting that with $\beta=0.5$ the $K_{2}$ branch did not disappear, but that instead its lower- $F$ bound was increased, and with little change near the high- $F$ bound. This suggests that the associated control mechanism is important in establishing the switch but has a secondary role in setting the strength of vortex shedding.

The lower- $F$ bound of the $K_{1}$ branch with $\beta=1$ and the upper- $F$ bound of the $K_{2}$ branch with $\beta=0.5$ were much the same as the values observed in figure 8 $(\beta=0)$. This suggests that the outer termination of those branches (i.e. the extent of the entrainment regime) does not depend on variations in the relative strength of motion-induced vorticity production, but is produced by some other mechanism.

At a fixed value of $F$, increasing oscillation amplitude leads to greater cylinder acceleration, hence increasing the motion-induced vorticity production. In turn this suggests that if the phase switch does occur as the result of a competition between pressure-gradient and motion-induced vorticity production mechanisms, the value of $F$ at which the switch occurs should reduce as $A$ increases. Exactly this effect can be seen in the experimental results of Stansby (1976), shown in figure 1.

While our suggestion seems a reasonable hypothesis for a control mechanism underlying the switch, it does not explain the detailed fluid mechanics of the phenomenon. Nor does it explain how the coupling of the wake to the cylinder motion comes about, or why $E$ changes from negative to positive along the $K_{1}$ solution branch as $F$ increases.

\section{Conclusions}

By studying an idealized two-dimensional flow past an oscillating circular cylinder at $R e=500$ we have been able to replicate the phase-switching behaviour observed in a number of experiments, and demonstrate that the switch is associated with a change in sign of mechanical energy transfer between the cylinder and the flow. We have also shown that additional unusual but periodic wakes can also exist, with hysteresis effects associated with the bifurcations to these states. This may help to explain some of the hysteresis behaviour observed in previous forced oscillation experiments.

The results suggest that the discontinuous switch in phase of vortex shedding results from the outcome of a competition between two vorticity production mechanisms. Simulations in which one of the production mechanisms was switched off resulted in the disappearance of one of the two periodic Kármán-mode solutions associated with the switch, supporting the idea that the switch does result from such a competition.

Our simulation results do not reproduce all of the observed behaviours that have been found in previous forced oscillation experiments, possibly due to the restriction to a single oscillation amplitude and Reynolds number, and also to the restriction to two

dimensions. The results do however suggest possible avenues for future experimental and three-dimensional numerical investigations.

A significant proportion of the simulations reported here was performed at the Australian National University Supercomputer Facility, and we thank its staff for their support and assistance. 


\section{REFERENCES}

Angrilli, F., Di Silvio, G. \& Zanardo, A. 1972 Hydroelasticity study of a circular cylinder in a water stream. In Flow-Induced Structural Vibrations IUTAM-IAHR Symposium, Karlsruhe (ed. E. Naudascher), pp. 504-512. Springer.

BARKLey, D. \& Henderson, R. D. 1996 Three-dimensional Floquet stability analysis of the wake of a circular cylinder. J. Fluid Mech. 322, 215-241.

Bearman, P. W. \& Currie, I. G. 1979 Pressure-fluctuation measurements on an oscillating circular cylinder. J. Fluid Mech. 91, 661-677.

Bishop, R. E. D. \& Hassan, A. Y. 1964 The lift and drag forces on a circular cylinder oscillating in a flowing fluid. Phil. Trans. R. Soc. Lond. A 277, 51-75.

Blackburn, H. M. \& Henderson, R. D. $1995 a$ Near-wake vorticity dynamics in bluff body flows. In 12th Australasian Fluid Mech. Conf., Sydney, pp. 17-20.

Blackburn, H. M. \& Henderson, R. D. $1995 b$ Wake dynamics in flow past an oscillating cylinder. In 9th Intl Conf. Numer. Meth. Laminar \& Turb. Flow, Atlanta, pp. 1479-1490.

Blackburn, H. M. \& Henderson, R. D. 1996 Lock-in behaviour in simulated vortex-induced vibration. Exptl Thermal Fluid Sci. 12, 184-189.

Blackburn, H. M. \& Karniadakis, G. E. 1993 Two- and three-dimensional simulations of vortexinduced vibration of a circular cylinder. In 3rd Intl Offshore \& Polar Engng Conf., Singapore, pp. 715-720.

Blackburn, H. M. \& Melbourne, W. H. 1997 Sectional lift forces for an oscillating cylinder in smooth and turbulent flows. J. Fluids Struct. 11, 413-431.

Brika, D. \& LANEville, A. 1993 Vortex-induced vibrations of a long flexible circular cylinder. J. Fluid Mech. 250, 481-508.

Den Hartog, J. P. 1934 The vibration problem in engineering. In Proc. 4th Intl Congr. Appl. Mech., pp. $36-53$.

FENG, C. C. 1968 The measurement of vortex-induced effects in flow past stationary and oscillating circular and D-section cylinders. Master's thesis, University of British Columbia.

Gerrard, J. H. 1966 The mechanics of the formation region of vortices behind bluff bodies. J. Fluid Mech. 25, 401-413.

Griffin, O. M. \& RAMberG, S. E. 1974 The vortex-street wakes of vibrating cylinders. J. Fluid Mech. 66, 533-576.

Gu, W., Chyu, C. \& Rockwell, D. 1994 Timing of vortex formation from an oscillating cylinder. Phys. Fluids 6, 3677-3682.

Henderson, R. D. 1995 Details of the drag curve near the onset of vortex shedding. Phys. Fluids 7, 2102-2104.

Henderson, R. D. 1997 Nonlinear dynamics and pattern formation in turbulent wake transition. J. Fluid Mech. 352, 65-112.

Henderson, R. D. \& KARNiADAKIS, G. E. 1995 Unstructured spectral element methods for simulation of turbulent flows. J. Comput. Phys. 122, 191-217.

Hornung, H. 1989 Vorticity generation and transport. In 10th Australasian Fluid Mech. Conf. KS3.1-7. Melbourne.

Karniadakis, G. E., Israeli, M. \& Orszag, S. A. 1991 High-order splitting methods for the incompressible Navier-Stokes equations. J. Comput. Phys. 97, 414-443.

Khalak, A. \& Williamson, C. H. K. 1996 Dynamics of a hydroelastic cylinder with very low mass and damping. J. Fluids Struct. 10, 455-472.

KhalaK, A. \& Williamson, C. H. K. 1997 Fluid forces and dynamics of a hydroelastic structure with very low mass and damping. J. Fluids Struct. 11, 973-982.

Koopmann, G. H. 1967 The vortex wakes of vibrating cylinders at low Reynolds numbers. J. Fluid Mech. 28, 501-512.

Lighthill, M. J. 1963 Boundary layer theory. In Laminar Boundary Layers (ed. L. Rosenhead), pp. 46-103. Oxford University Press.

Lu, X.-Y. \& Dalton, C. 1996 Calculation of the timing of vortex formation from an oscillating cylinder. J. Fluids Struct. 10, 527-541.

Meneghini, J. R. \& Bearman, P. W. 1995 Numerical simulation of high amplitude oscillatory flow about a circular cylinder. J. Fluids Struct. 9, 435-455. 
Morton, B. R. 1984 The generation and decay of vorticity. Geophys. Astrophys. Fluid Dyn. 28, 277-308.

Novak, M. \& Tanaka, H. 1975 Pressure correlations on a vibrating cylinder. In 4th Intl Conf. Wind Effects Build. \& Struct., Heathrow, pp. 227-232. Cambridge University Press.

OngOREN, A. \& Rockwell, D. 1988 Flow structure from an oscillating cylinder. Part 1. Mechanisms of phase shift and recovery in the near wake. J. Fluid Mech. 191, 197-223.

Orszag, S. A., Israeli, M., \& Deville, M. O. 1986 Boundary conditions for incompressible flows. J.Sci. Comput. 1, 75-111.

Parkinson, G. 1989 Phenomena and modelling of flow-induced vibrations of bluff bodies. Prog. Aerospace Sci. 26, 169-224.

Perry, A. E., Chong, M. S. \& Lim, T. T. 1982 The vortex-shedding process behind two-dimensional bluff bodies. J. Fluid Mech. 116, 77-90.

Shariff, K., Pulliam, T. H. \& Ottino, J. M. 1991 A dynamical systems analysis of kinematics in the time-periodic wake of a circular cylinder. In Lectures in Applied Mathematics, vol. 28 (ed. Anderson \& Greengard), pp. 613-646. AMS.

StANSBY, P. K. 1976 The locking-on of vortex shedding due to the cross-stream vibration of circular cylinders in uniform and shear flows. J. Fluid Mech. 74, 641-655.

ToEBES, G. H. 1969 The unsteady flow and wake near an oscillating cylinder. Trans. ASME J. Basic Engng 91, 493-505.

Wieselsberger, C. 1921 Neure festellungen über die gesetze des flüssigkeit- und luftwiderstands. Phys. Z. 22, 321-328.

WiLliamson, C. H. K. 1989 Oblique and parallel modes of vortex shedding in the wake of a circular cylinder at low Reynolds numbers. J. Fluid Mech. 206, 579-627.

Williamson, C. H. K. \& Roshko, A. 1988 Vortex formation in the wake of an oscillating cylinder. J. Fluids Struct. 2, 355-381.

ZdRaVkovich, M. M. 1982 Modification of vortex shedding in the synchronization range. Trans. ASME J. Fluids Engng 104, 513-517. 